\title{
SUR LA THÉORIE DES VARIÉTÉS ALGÉBRIQUES
}

\author{
C. CHEVALLEY
}

\section{Introduction}

A. Weil a généralisé la notion de variété algébrique d’un espace affine (ou projectif) en introduisant la notion de variété abstraite (nous dirons simplement "variété" au lieu de "variété abstraite," appelant variétés affines les variétés algébriques d'un espace affine). La définition donnée par A. Weil des variétés algébriques s'inspire manifestement de la notion de variété différentiable, conçue comme réunion d'un nombre fini de morceaux dont chacun peut être considéré comme une partie ouverte d'un espace numérique, les variétés des espaces affines se substituant aux ensembles ouverts des espaces euclidiens. Il subsiste cependant une différence notable entre la notion de variété différentiable et celle de variété algébrique au sens de A. Weil: dans une variété différentiable, il n'existe aucun système de coordonnées privilégié, et la même variété différentiable peut se représenter de bien des manières différentes comme réunion de morceaux euclidiens; au contraire, une variété au sens de Weil se trouve munie d'une décomposition uniquement determinée en variétés affines, et chacune de ces variétés affines est plongée d'une manière bien déterminée dans un espace affine, ce qui signifie qu'elle est munie d'un système de coordonnées privilégié.

Ceci étant, il peut paraître désirable de pousser plus loin l'analogie entre variétés différentiables et variétés algébriques en cherchant à définir ces dernières de manière invariante par rapport aux changements de coordonnées. Le problème peut se formuler ainsi. Appelons invariant d'une variété $V$ tout objet $M(V)$ intrinsèquement attaché à une variété $V$ quelconque et tel que, si deux variétés $V, V^{\prime}$ sont en correspondance birationnelle partout birégulière ${ }^{1)}$ les objets $M(V)$ et $M\left(V^{\prime}\right)$ attachés à $V$ et à $V^{\prime}$ soient isomorphes (nous supposons que ces "objets" sont des ensembles munis de structures convenables). Le problème est alors de déterminer un invariant $M(V)$ tel que, réciproquement,

Received July 2, 1954.

1) Nous entendons par là qu'il y a entre $V$ et $V^{\prime}$ une correspondance birationnelle telle qu'à tout point de $V$ corresponde un point de $V^{\prime}$ et réciproquement, la correspondance étant de plus birégulière. 
deux variétés $V$ et $V^{\prime}$ pour lesquelles $M(V)$ et $M\left(V^{\prime}\right)$ sont isomorphes soient en correspondance birationnelle partout birégulière. Il résulte implicitement des travaux de $M$. Zariski que ce problème peut se résoudre de la manière suivante. Pour chaque point $P$ de $V$, l'ensemble des fonctions sur $V$ qui sont définies en $P$ est un sous-anneau $\mathfrak{o}(P)$ du corps des fonctions sur $V$; l'ensemble $M(V)$ de tous les anneaux $\mathfrak{b}(P)$, pour tous les $P \in V$, possède alors les propriétés requises d'invariance: c'est ce que nous démontrons explicitement dans la première partie. Nous appelons l'ensemble $M(V)$ le modèle de la variété $V$. De plus, nous caractérisons les ensembles de sous-anneaux d'une extension de type fini du domaine fondamental qui sont des modèles de variétés. Il résulte notamment de cette caractérisation que toute variété est birationnellement équivalente (au moyen d'une correspondance partout birégulière) à une variété qui est définie au moyen de variétés affines mais dépourvues de frontières.

Ceci fait, nous proposons une nouvelle définition, entièrement invariante, de la notion de variété. On peut à vrai dire objecter à cette définition qu'on y perd la notion du corps de définition d'une variété ainsi que celle de point générique par rapport à un corps de définition. De fait, ces notions sont incompatibles avec la condition d'invariance que nous nous sommes imposée. En effet, il est bien clair qu'il n'y a aucune relation nécessaire entre les corps de définition de deux variétés $V$ et $V^{\prime}$ qui sont birationnellement équivalentes; et, si $K$ est un corps de définition commun pour ces deux variétés, un point de $V$ peut être générigue par rapport à $K$ alors que son correspondant dans $V^{\prime}$ est rationnel sur $K$ : c'est ce que montre déjà l'exemple simple où $V, V^{\prime}$ sont des droites convenables d'un plan affine. Si l'on admet que l'objet de la géométrie algébrique est l'étude des propriétés des variétés qui sont invariantes par les transformations birationnelles (soit que l'on admette toutes les transformations birationnelles soit que l'on se limite à celles qui sont partout birégulières), il peut paraitre fâcheux, au moins d'un point de vue esthétique, de faire jouer à la notion non invariante de corps de définition un rôle aussi important que celui que lui fait jouer A. Weil. Par contre, il est indiscutable que la notion de point générique par rapport à un corps de définition $K$, dont nous critiquons le rôle dans la définition d'une variété, se révèle d'une utilité technique considérable. Pour pouvoir en disposer dans la définition que nous proposons, nous introduisons la notion de $K$-variété, $K$ étant un sous-corps du domaine fondamental 
$\Omega$ sur lequel $\Omega$ est de degré de transcendance infini. Toute variété peut être munie de structures de $K$-variétés pour tous les sous-corps suffisamment vastes du domaine universel; mais, même une fois $K$ fixé, une même variété $V$ peut être munie de plusieurs structures différentes de $K$-variété. La notion de point générique par rapport à $K$ (ou à un sur-corps de $K$ ) est définie pour les $K$ variétés et possède les propriétés usuelles.

Une autre divergence entre la définition de A. Weil et la nôtre est que nous revenons au point de vue "ensembliste" classique, duquel une variété est l'ensemble de ses points. Il nous semble que le langage de la théorie des ensembles, autant et même peut-être plus que le langage géométrique, constitue une aide précieuse à la compréhension de la géométrie algébrique. La raison pour laquelle A. Weil s'est cru forcé de renoncer aux facilités de ce langage, à savoir que la projection (au sens de la théorie des ensembles) d'une sousvariété d'un produit $E \times F$ de deux variétés sur $E$ (ou sur $F$ ) n'est pas nécessairement une sous-variété, nous a paru bien mince en proportion au sacrifice imposé. Rien n'empêche d'introduire un mot tel que "projection fermée" ou “projection algébrique” pour désigner ce que A. Weil appelle projection. Nous appelons constructibles les sous-ensembles d'une variété qui peuvent s'obtenir à partir des sous-variétés par les opérations suivantes: prendre le complémentaire d'un ensemble; prendre la réunion ou l'intersection de deux ensembles. Les projections d'une partie constructible du produit de deux variétés sur les facteurs du produit sont alors constructibles, ce qui montre que la classe des ensembles constructibles est fermée relativement aux opérations les plus importantes que l'on ait à faire en géométrie algébrique. A ce sujet, nous voudrions faire l'observation suivante. Soit $\mathfrak{P}$ une propriété que peuvent ou non posséder les points d'une variété $E$. On peut attribuer (au moins) deux significations précises distinctes à la phrase vague "les points de $E$ possèdent en général la propriéte $\mathfrak{B}$," à savoir les suivantes: 1) tout point de $E$ qui est générique par rapport à un corps convenable possède la propriété $\mathfrak{P} ; 2$ ) l'ensemble des points qui possèdent la propriété $\mathfrak{P}$ contient une partie ouverte non vide (au sens de la topologie de Zariski) de $E$, c'est-à-dire: il existe un nombre fini de sousvariétés de $E$, de dimensions inférieures à celle de $E$, telles que tout point de $E$ n'appartenant à aucune de ces șous-variétés possède la propriété $\mathfrak{P}$. La condition 2) entraine manifestement 1 ), mais la réciproque n'est pas vraie. Il est 
souvent beaucoup plus facile d'établir 1) que d'établir 2) (par exemple, il est bien plus facile d'établir qu'une section générique d'une variété normale par une hypersurface est normale que de montrer que "presque toutes" ces sections sont normales). Néanmoins, il nous semble qüil y a lieu d'établir, toutes les fois que c'est possible, que la condition plus forte 2) est satisfaite. En effet, si $K$ est un corps tel que $E$ possède "assez" de points rationnels sur $K$, la condition 2) entraine qu'il existe un point rationnel sur $K$ qui possède la propriété $\mathfrak{P}$, alors qu'il n'en est pas ainsi de la condition 1). Notons en passant que, si l'ensemble des points qui possèdent la propriété $\mathfrak{B}$ est constructible et si la condition 1) est satisfaite, il en est de même de 2).

Nous avons cru devoir nous écarter de la terminologie de A. Weil sur un autre point encore, à savoir en ce qui concerne la définition des sous-variétés. Il nous paraît en effet indésirable de refuser ce nom aux analogues pour les variétés algébriques des sous-variétés ouvertes des variétés différentiables. Pour prendre un exemple, dans la terminologie de A. Weil, un espace projectif est une variété $P$, l'espace affine qu'on en déduit par ablation d'un plan à l'infini est également une variété $S$, mais $S$ n'est pas une sous-variété de $P$. Nous proposons donc d'appeler sous-variétés de $E$ toutes les variétés qui sont des parties relativement ouvertes (au sens de la topologie de Zariski) de sous-variétés de $E$ au sens de A. Weil.

Les démonstrations que nous faisons dans les parties II, III, IV sont peuvent être lues sans connaitre la théorie des variétés de A. Weil (sauf celle par laquelle nous établissons au début de II l'équivalence entre notre définition et celle de $\mathrm{A}$. Weil). Les résultats que nous y établissons sont en partie des résultats plus ou moins connus de tous les spécialistes en géométrie algébrique, mais qui n'avaient pas encore été formulés explicitement (à notre connaissance du moins), en partie des résultats déjà établis par A. Weil dans sa théorie des variétés. En ce qui concerne ces derniers, si nous en avons donné des démonstrations, ce n'est pas avec l'illusion de présenter dans les pages qui suivent une exposition complète de la théorie à partir des définitions que nous proposons, mais plutôt pour montrer in concreto sur quelques exemples la manière dont fonctionnent les notions que nous avons introduites. 
I.

Nous appellerons variétés les variétés abstraites de A. Weil, et variétés affines les variétés de A. Weil. Nous désignerons par $\Omega$ un domaine universel fixe (au sens de A. Weil).

Soit $V$ une variété: l'ensemble, que nous désignerons par $\Re(V)$, des fonctions sur $V$ est alors muni d'une structure de $\operatorname{corps}^{2)}$; les fonctions constantes forment un sous-corps de $\Re(V)$ canoniquement isomorphe à $Q$ et que nous identifierons à $\Omega$, tout élément $a \in \Omega$ étant identifié à la fonction constante de valeur $a$.

Soient $K$ un corps de définition de $V$ et $P$ un point générique de $V$ par rapport à $K$. L'ensemble des fonctions $f$ sur $V$ qui sont définies sur $K$ est alors un sous-corps de $\Re(V)$, soit $\Re_{K}(V)$, et l'application $f \rightarrow f(P)$ est un $K$-isomorphisme (i.e. un isomorphisme laissant les éléments de $K$ fixes) de $\Re_{K}(V)$ sur $K(P)$. Le corps $\mathfrak{A}(V)$ est la réunion des corps $\mathfrak{A}_{K}(V)$ pour tous les corps de définition $K$ de $V$. Si $K^{\prime}$ est un corps de définition de $V$ contenant $K$, et $P$ un point générique de $V$ par rapport à $K^{\prime}$, le corps $K^{\prime}(P)$ est engendré par $K^{\prime}$ et $K(P)$, et les extensions $K(P) / K, K^{\prime} / K$ sont linéairement disjointes sur $K$; ces assertions se déduisent immédiatement du fait qu'elles sont valables pour les variétés affines.

Supposons la variété $V$ donnée par des variétés affines $V_{\alpha}$, des correspondances birationnelles $T_{\beta \alpha}$ et des frontières $F_{\alpha}$. Soit $n(\alpha)$ la dimension de l'espace affine dans lequel se trouve la variété $V_{\alpha}$; soient $K$ un corps de définition de $V$ et $P_{\alpha}$ un point générique de $V_{\alpha}$ par rapport à $K$. Soient $x_{i}$ les coordonnées de $P_{\alpha}(1 \leqq i \leqq n(\alpha))$. Le point $P_{\alpha}$ est un représentant d'un point générique $P$ de $V$ sur $K . \quad$ Il existe des fonctions $f_{i}$ sur $V$ telles que $f_{i}(P)=x_{i}$; ces fonctions ne dépendent que de $V_{\alpha}$, non des choix de $K$ et de $P_{a}$. En effet, si $P^{\prime}=\left(x_{1}^{\prime}\right.$, $\left.\ldots, x_{n(\alpha)}^{\prime}\right)$ est un autre point générique de $V$ sur $K, P^{\prime}$ est une spécialisation (générique) de $P$ par rapport à $K$, d'où $f_{i}\left(P^{\prime}\right)=x_{i}^{\prime}$, ce qui montre que, pour $K$ donné, les $f_{i}$ ne dépendent pas du choix de $P$. Pour montrer que les $f_{i}$ ne changent pas si on remplace $K$ par un autre corps de définition $K^{\prime}$, il suffit de prendre pour $P_{\alpha}$ un point de $V_{\alpha}$ qui est générique aussi bien par rapport à $K$ que par rapport à $K^{\prime}$. Les fonctions $f_{i}$ sont appelées les fonctions coordonnées relatives à $V_{\alpha}$; l'anneau $\Omega\left[f_{1}, \ldots, f_{n(\alpha)}\right]$ s'appelle, lanneau affine relatif à $V_{\alpha}$. D'une manière générale, nous appellerons anneaus affine du corps $\Re(V)$ tout

2) Ce n'est pas un sous-corps du domaine universel; c'est donc un corps abstrait dans la terminologie de A. Weil. 
sous-anneau de ce corps contenant $\Omega$, engendré par un nombre fini d'éléments sur $\Omega$ et dont le corps des quotients est $\mathfrak{R}(V)$.

Lorsque $V$ est une variété affine, nous pourrons parler des fonctions coordonnées sur $V$ et de l'anneau affine de $V$ sans donner de précision supplémentaire.

Revenant aux notations utilisées plus haut, il est clair qu'il existe pour chaque $\alpha$ un isomorphisme bien determiné du corps $\Re\left(V_{\alpha}\right)$ sur $\Re(V)$, soit $\theta_{\alpha}$, qui peut se définir comme suit: si $f_{\alpha}$ est une fonction sur $V_{\alpha}$, définie sur un corps de définition $K$ de $V$, et $P_{\alpha}$ un point générique de $V_{\alpha}$ par rapport à $K$, représentant d'un point $P$ de $V, \theta_{\alpha}\left(f_{\alpha}\right)$ prend en $P$ la valeur $f_{\alpha}\left(P_{\alpha}\right)$. L'isomorphisme $\partial_{\alpha}$ fait correspondre aux fonctions coordonnées sur $V_{\alpha}$ les fonctions coordonnées sur $V$ relatives à $V_{\alpha}$.

Soient maintenant $U$ et $V$ des variétés, et $T$ une correspondance birationnelle entre $U$ et $V$. Il correspond alors à $T$ un isomorphisme $T^{*}$ de $\mathfrak{A}(U)$ sur $\Re(V)$, qui se définit de la manière suivante. Soient $f$ un élément de $\mathfrak{R}(U), K$ un corps de définition commun pour $U, V, f$ et $T$ et $P$ un point générique de $U$ par rapport à $K$. Il y a alors un point $Q$ et un seul de $V$ tel que $P \times Q$ soit dans $T$, et $Q$ est générique sur $V$ par rapport à $K$ (cela résulte immédiatement de $[\mathrm{W}], \mathrm{IV}, 7$, th. 16). Il y a donc une fonction $g$ sur $V$, définie sur $K$, telle que $g(Q)=f(P)$. Cette fonction ne dépend pas des choix de $K$ et de $P$. Il suffit de montrer que $g$ n'est pas changée si on remplace $K$ par un corps de définition $K^{\prime}$ contenant $K$ et $P$ par un point générique $P^{\prime}$ de $U$ par rapport à $K^{\prime}$. Or, si $Q^{\prime}$ est le point tel que $P^{\prime} \times Q^{\prime}$ soit dans $T, P \times Q$ est spécialisation générique de $P^{\prime} \times Q^{\prime}$ par rapport à $K$, d'où il résulte tout de suite que l'égalité $g(Q)=f(P)$ entraîne $g\left(Q^{\prime}\right)=f\left(P^{\prime}\right)$. Nous poserons $g=T^{*}(f)$; on vérifie tout de suite que l'application $T^{*}$ ainsi définie est un isomrphisme de $\mathfrak{R}(U)$ sur $\mathfrak{R}(V)$. Si $P_{1} \times Q_{1}$ est un point de $T$ tel que $f$ soit défini en $P_{1}$ et $g$ défini en $Q_{1}$, on a $f\left(P_{1}\right)=g\left(Q_{1}\right)$, comme il résulte tout de suite du fait qu'avec les notations utilisées ci-dessus $P_{1} \times Q_{1}$ est une spécialisation de $P \times Q$ par rapport à $K$.

A tout point $P$ d'une variété $V$, on peut associer un sous-anneau $\mathfrak{D}(P)$ du corps $\mathfrak{A}(V)$, à savoir l'anneau des fonctions sur $V$ qui sont définies en $P$ et y prennent des valeurs finies. Supposons la variété $V$ donnée au moyen de variétés affines $V_{\alpha}$, de frontières $F_{\alpha}$ et de correspondances birationnelles $T_{\beta \alpha}$. Soit $\alpha$ un indice tel que $P$ ait un représentant $P_{\alpha}$ dans $V_{\alpha}$. L'anneau $\mathfrak{o}(P)$ contient alors évidemment l'anneau affine $\mathfrak{B}_{\alpha}$ relatif à $V_{\alpha}$. De plus, les fonctions de $\mathfrak{B}_{\alpha}$ 
qui prennent la valeur 0 en $P$ forment un idéal maximal $\mathfrak{p}_{\alpha}$ de $\mathfrak{B}_{\alpha}$. et $\mathfrak{o}(P)$ contient l'anneau local $\mathfrak{D}^{\prime}$ de $\mathfrak{p}_{\alpha}$ (i.e. l'ensemble des fig, où $f, g$ sont dans $\mathfrak{V}_{\alpha}$ mais où $g$ n'est pas dans $p_{\alpha}$ ). On a d'ailleurs $o(P)=0^{\prime}$. Soient en effet $K$ un corps de définition pour $V$ et pour une fonction $f \in o(P)$ et $Q$ un point générique de $V$ par rapport à $K$. Soient $f_{1}, \ldots, f_{n(\alpha)}$ les fonctions coordonnées relatives à $V_{\alpha}, y_{1}, \ldots, y_{n(\alpha)}$ les coordonnées du représentant $Q_{\alpha}$ de $Q$ dans $V_{\alpha}$ et $x_{1}, \ldots$, $x_{n(\alpha)}$ les coordonnées de $P_{\alpha}$. Puisque $f$ est définie en $P, f(Q)$ appartient à l'anneau de spécialisation de $P$ dans $K(Q)$, ce qui montre que $f(Q)$ est de la forme $A\left(y_{1}, \ldots, y_{n}\right) B^{-1}\left(y_{1}, \ldots, y_{n}\right)$ où $A, B$ sont des polynômes à coefficients dans $K$ tels que $B\left(x_{1}, \ldots, x_{n}\right) \neq 0$. Il en résulte que l'on a $f=A\left(f_{1}, \ldots, f_{n}\right) B^{-1}\left(f_{1}\right.$, $\left.\ldots, f_{n}\right)$, d'où $\mathfrak{o}(P)=\mathfrak{v}^{\prime}$. Nous appellerons $\mathfrak{o}(P)$ l'anneau local du point $P$. C'est un "anneau local” au sens technique du terme; son idéal maximal se compose des fonctions sur $V$ qui prennent la valeur 0 en $P$.

Proposition 1. Soient $T$ une correspondance birationnelle entre une variété $U$ et une variété $V, T^{*}$ l'isomorphisme correspondant de $\mathfrak{R}(U)$ sur $\mathfrak{N}(V), P$ un point de $U$ et $Q$ un point de $V$. Pour que $P \times Q$ appartienne $\grave{a} T$, il faut et suffit que la condition suivante soit satisfaite: si on désigne par p et q les idéaux maximaux des anneaux locaux $\mathfrak{o}(P)$ et $\mathfrak{o}(Q)$ de $P$ et de $Q$, lidéal engendré par $T^{*}(\mathfrak{p})$ et q dans l'anneau engendré par $T^{*}(\mathfrak{o}(P))$ et $\mathfrak{o}(Q)$ ne contient pas 1 .

Les projections de $T$ sur $U$ et sur $V$ sont des correspondances birationnelles; les inverses de ces correspondances définissent des isomorphismes $\pi_{V}$ et $\pi$ r de $\mathfrak{A}(U)$ et $\mathfrak{R}(V)$ sur $\mathfrak{R}(T)$. Supposons d'abord que $P \times Q$ appartienne à T. Il est clair que, si $f$ est une fonction sur $U$ définie en $P, \pi_{U}(f)$ est définie en $P \times Q$; de même, si une fonction $g$ sur $V$ est définie en $Q, \pi r(g)$ est définie en $P \times Q$. De plus, si $f \in \mathfrak{p}, \pi_{C^{\prime}}(f)$ appartient à l'idéal maximal $\mathfrak{r} \operatorname{de} \mathfrak{o}(P \times Q)$, puisque $\left(\pi_{U}(f)\right)(P \times Q)=f^{*}(P)$; de même, si $g \in \mathfrak{q}$, on a $\pi_{r}(g) \in$ r. L'idéal engendré par $\pi_{r}(\mathfrak{p})$ et $\pi_{r} \cdot(\mathfrak{q})$ dans $\mathfrak{p}(P \times Q)$ est donc contenu dans $\mathfrak{r}$. Comme on a $\pi_{C}=\pi_{1} \circ T^{*}$, on voit que la condition énoncée est nécessaire. Supposons la maintenant réciproquement satisfaite. Supposons les variétés $U$ et $V$ données par des variétés affines $U_{\alpha}, V_{\gamma}$, des frontières sur ces variétés affines et des correspondances birationnelles. Supposons que $P$ admette un représentant $P_{\alpha}$ dans $U_{\alpha}$ et $Q$ un représentant $Q_{r}$ dans $V_{r}$. Nous identifierons $\Re\left(U_{\alpha}\right)$ à $\Re(U)$ au moyen de l'isomorphisme qui fait correspondre les fonctions coordonnées relatives à $U_{\alpha}$ aux fonctions coordonnées sur $U_{\alpha}$; et de même pour $\mathfrak{i}\left(V_{\Upsilon}\right)$ 
et $\Re(V)$. La correspondance birationnelle $T$ donne une correspondance birationnelle $T_{\curlyvee \alpha}$ entre $U_{\alpha}$ et $V_{\curlyvee}$; l'isomorphisme de $\Re\left(U_{\alpha}\right)$ sur $\Re\left(V_{\Upsilon}\right)$ associé à $T_{\Upsilon \alpha}$ est encore $T^{*}$ (grâce à nos identifications). De plus, on a $\mathfrak{D}(P)=\mathfrak{D}\left(P_{\alpha}\right), \mathfrak{D}(Q)=$ $\mathfrak{b}\left(Q_{\Upsilon}\right)$. Soient $\mathfrak{U}_{\alpha}$ l'anneau affine de $U_{\alpha}$ et $\mathfrak{B}_{\curlyvee}$ celui de $V_{\Upsilon}$. Nous identifierons encore $\mathfrak{R}\left(T_{\gamma_{\alpha}}\right)$ à $\mathfrak{R}(T)$ au moyen de l'isomorphisme qui fait correspondre les fonctions coordonnées relatives à $T_{r \alpha}$ aux fonctions coordonnées sur $T_{\text {ra. Nous }}$ allons montrer que l'anneau affine $\mathfrak{I}$ de $T_{\Upsilon \alpha}$ est identique à l'anneau engendré par $\pi_{U}\left(\mathfrak{l}_{\alpha}\right)$ et $\pi_{r}\left(\mathfrak{B}_{\curlyvee}\right)$. Les variétés $U_{\alpha}$ et $V_{\curlyvee}$ sont dans des espaces affines; $T_{\gamma \alpha}$ est une sous-variété du produit de ces espaces affines dont les projections sur les deux facteurs sont $U_{\alpha}$ et $V_{\curlyvee}$ respectivement. Si $f_{1}, \ldots, f_{m_{(\alpha)}}$ et $g_{1}, \ldots$ ., $g_{n(r)}$ sont les fonctions coordonnées sur $U_{\alpha}$ et sur $V_{r}$, les fonctions coordonnées sur $T_{\gamma_{\alpha}}$ sont donc les $\pi_{c}\left(f_{i}\right)$ et les $\pi_{v}\left(g_{j}\right)$, ce qui démontre notre assertion. Puisque $\pi_{c}=\pi_{r} \circ T^{*}$, il résulte de notre hypothèse que l'idéal engendré par $\mathfrak{p}^{\prime}$ $=\pi_{c}(\mathfrak{p})$ et $q^{\prime}=\pi_{V}(\mathfrak{q})$ dans l'anneau $z$ engendré par $\pi_{c}(\mathfrak{D}(P))$ et $\pi_{V}(\mathfrak{D}(Q))$ ne contient pas 1 . Cet idéal est donc contenu dans au moins un idéal maximal $\mathfrak{r}$ de 马. Soit $\varphi$ l'homomorphisme canonique de $\xi$ sur $\xi / \mathfrak{r}$; identifiant $\Omega$ dे un souscorps de $\& / \mathfrak{r}, \varphi$ coïncide avec l'identité sur $\Omega$. On a $f_{i} \equiv f_{i}(P)(\bmod \mathfrak{p})$, d'où $\pi_{U}\left(f_{i}\right) \equiv f_{i}(P)\left(\bmod \mathfrak{p}^{\prime}\right)$, et de même $\pi_{r}\left(g_{j}\right) \equiv g_{j}(Q)\left(\bmod q^{\prime}\right)$. On en déduit que $\varphi\left(\pi_{U}\left(f_{i}\right)\right)=f_{i}(P), \varphi\left(\pi_{r}\left(g_{j}\right)\right)=g_{j}(Q)$. On a d'ailleurs $f_{i}(P)=f_{i}\left(P_{a}\right), g_{j}(Q)$ $=g_{j}\left(Q_{r}\right)$. Comme $\varphi$ induit un homomorphisme de $\mathfrak{I}$, on voit qu'il y a un homomorphisme de $\mathfrak{I}$ dans $\Omega$ qui coïncide avec l'identité sur $\Omega$ et qui applique les fonctions coordonnées sur $T_{\Upsilon \alpha}$ sur les coordonnées du point $P_{\alpha} \times Q_{\text {r. }} \quad \mathrm{Ce}$ dernier appartient donc à $T_{\text {ra }}$, ce qui montre que $P \times Q$ appartient à $T$.

Corollaire. Soient $P, Q$ des points distincts d'une variété $V$, et $\mathfrak{p}$, q les idéaux maximaux de leurs anneaux locaux $\mathrm{b}(P)$ et $\mathrm{o}(Q)$. L'idéal engendré par $\mathfrak{p}$ et $\mathfrak{q}$ dans l'anneau engendré par $\mathfrak{D}(P)$ et $\mathfrak{o}(Q)$ contient alors 1 .

Il suffit d'appliquer la prop. 1 au cas où $U=V$ et où $T$ est la diagonale de $V \times V$.

Soit $V$ une variété donné par des variétés affines $V_{\alpha}$, des frontières $F_{\alpha}$ sur les $V_{\alpha}$ et des correspondances birationnelles entre les variétés $V_{\alpha}$. Soit $\mathfrak{B}_{\alpha}$ l'anneau affine relatif à $V_{\alpha}$, et soit $M_{\alpha}$ l'ensemble des anneaux locaux des points de $V$ qui ont des représentants dans $V_{\alpha}$. Nous identifions $\mathfrak{B}_{\alpha}$ à l'anneau affine de $V_{\alpha}$. Les fonctions de $\mathfrak{B}_{\alpha}$ qui sont nulles en tout point de $F_{\alpha}$ forment un idéal $\mathfrak{f}_{\alpha}$. Il est clair que $M_{\alpha}$ est l'ensemble des anneaux locaux des idéaux 
maximaux de $\mathfrak{B}_{\propto}$ qui ne contiennent pas $\mathfrak{f}_{\alpha}$.

D'une manière générale, si $\Re / \Omega$ est une extension de type fini de $\Omega$, nous appellerons anneau affine de $\mathfrak{R}$ tout sous-anneau de $\mathfrak{R}$ contenant $\Omega$, de la forme $\Omega\left[x_{1}, \ldots, x_{n}\right]$ (où les $x_{i}$ sont dans $\Re$ ) et admettant $\Re$ comme corps des quotients. Nous appellerons localité de $\mathfrak{R}$ tout sous-anneau de $\mathfrak{R}$ qui est anneau local d'un idéal maximal d'un anneau affine de $\mathfrak{R}$. Si $\mathfrak{B}$ est un anneau affine de $\mathfrak{R}$, nous appellerons modèle affine attaché à $\mathfrak{R}$, et nous désignerons par $M(\mathfrak{B})$, l'ensemble des anneaux locaux des idéaux maximaux de $\mathfrak{B}$. Tout ensemble de localités qui peut se mettre sous la forme $M(\mathfrak{B})$ pour un certain anneau affine $\mathfrak{B}$ sera appelé un modèle affine. Soient $\mathfrak{D}, \mathfrak{D}^{\prime}$ des localités, et $\mathfrak{p}, \mathfrak{p}^{\prime}$ leurs idéaux maximaux; nous dirons que $D$ et $D^{\prime}$ se correspondent si l'idéal engendré par $\mathfrak{p}$ et $\mathfrak{p}^{\prime}$ dans l'anneau engendré par $\mathfrak{o}$ et $\mathfrak{b}^{\prime}$ ne contient pas 1 . Nous dirons qu'un ensemble non vide $M$ de localités de $\mathfrak{R}$ est un modèle (du corps $\Re$ ) si les conditions suivantes sont satisfaites: a) deux localités distinctes de l'ensemble $M$ ne se correspondent jamais; b) $M$ est la réunion d'un nombre fini de modèles affines. Notre terminologie n'est pas contradictoire, car tout modèle affine de $\mathfrak{R}$ est un modèle de $\mathfrak{R}$ au sens que nous venons de définir. Soient en effet $\mathfrak{o}$ et $\mathfrak{v}^{\prime}$ les anneaux locaux de deux idéaux maximaux distincts $\mathfrak{M}$ et $\mathfrak{M}$ d'un anneau affine $\mathfrak{B}$. Puisque $\mathfrak{M}$ est maximal, $\mathfrak{M}+\mathfrak{M}^{\prime}$ contient 1 , et il en résulte immédiatement que $\mathfrak{D}$ et $\mathfrak{v}^{\prime}$ ne se correspondent pas.

Établissons maintenant le

Lemme 1. Soient $\mathfrak{B}$ un anneau affine de $\mathfrak{R}$, et $\{$ un idéal $\neq\{0\}$ de $\mathfrak{B}$. L'ensemble $M$ des anneaux locaux de ceux des idéaux maximaux de $\mathfrak{B}$ qui ne contiennent pas f est alors un modèle de $\mathfrak{R}$.

Il est clair que $f$ admet un ensemble fini de générateurs $x_{1}, \ldots, x_{h}$ tous $\neq 0$. Soit $\mathfrak{B}_{i}=\mathfrak{B}\left[x_{i}^{-1}\right]$; chaque $\mathfrak{W}_{i}$ est manifestement un anneau affine de $\Re$. Soient $\mathfrak{M}_{i}$ un idéal maximal de $\mathfrak{W}_{i}$, o son anneau local, $\mathfrak{p}=\mathfrak{M}_{i}$ l'idéal maximal de $\mathfrak{D}$ et $\mathfrak{M}$ l'idéal premier $\mathfrak{p} \cap \mathfrak{V}$ de $\mathfrak{B}$. Puisque $\mathfrak{M}_{i}$ est maximal, $\mathfrak{M}_{i} / \mathfrak{M}_{i}=\Omega$; il en résulte que $\mathfrak{o} / \mathfrak{p}=\Omega$; on a $\Omega \subset \mathfrak{B} / \mathfrak{M} \subset \mathfrak{o} / \mathfrak{p}=\Omega$, d'où $\mathfrak{B} / \mathfrak{M}=\Omega$, et $\mathfrak{R}$ est un idéal maximal de $\mathfrak{B}$. Tout élément de $\mathfrak{D}$ non contenu dans $\mathfrak{p}$ étant inversible dans $\mathfrak{D}$, il est clair que l'anneau local $\mathfrak{n}^{\prime}$ de $\mathfrak{M}$ est contenu dans $\mathfrak{0}$. Tout élément de $\mathfrak{W}_{i}$ peut se mettre sous la forme $x_{i}^{-e} y$, avec $e>0, y \in \mathfrak{B}$, et tout élément de $\mathfrak{D}$ se met sous la forme $u v^{-1}$, où $u, v$ sont dans $\mathfrak{B}_{i}, v \notin \mathfrak{M}_{i}$; écrivant $u=x_{i}^{-e} y, v=x_{l}^{-f} z$, avec $y, z \in \mathfrak{B}$, on a $u v^{-1}=x_{i}^{f-e} y z^{-1}$. Puisque $x_{i}^{-1} \in \mathfrak{S}_{i}, x_{i}$ n'est pas dans $\mathfrak{D i}_{2}$, ni 
par suite dans $\mathfrak{M}$, et $z=x_{i}^{f} v \notin \mathfrak{M} \mathfrak{R}_{i}$, d'où $z \notin \mathfrak{N}$; il en resulte que $u v^{-1} \in \mathrm{o}^{\prime}$, d'où $\mathfrak{v}^{\prime}=\mathfrak{v}$. Le modèle affine attaché à $\mathfrak{W}_{i}$ est donc contenu dans $M$. Soient réciproquement $\mathfrak{o}$ un élément de $M$, anneau local d'un idéal maximal $\mathfrak{D}$ de $\mathfrak{B}$ qui ne contient pas $\mathfrak{i}$. Il y a alors un $i$ tel que $x_{i} \neq \mathfrak{M}$, d'où $x_{i}^{-1} \in \mathfrak{D}, \mathfrak{W}_{i} \subset \mathfrak{o}$. Si $\mathfrak{p}=$ $\mathfrak{M}_{0}$ est l'idéal maximal de $\mathfrak{n}$, et $\mathfrak{M}_{i}=\mathfrak{p} \cap \mathfrak{X}_{i}$, on a $\Omega \subset \mathfrak{W}_{i} / \mathfrak{M}_{i} \subset \mathfrak{p} / \mathfrak{p}=\Omega$, et $\mathfrak{M}_{i}$ est un idéal maximal. On voit tout de suite que $\mathfrak{o}$ est son anneau local. L'ensemble $M$ est donc la réunion des modèles affines attachés aux anneaux $\mathfrak{W}_{i}$, ce qui démontre le lemme 1.

Proposition 2. Si $V$ est une variété, l'ensemble des anneaux locaux des points de $V$ est un modèle du corps des fonctions sur $V$.

Cela résulte immédiatement du lemme 1 et de ce qui a été dit plus haut.

Nous dirons que l'ensemble des anneaux locaux des points de $V$ est le modèle de la variété $V$.

Soient $\mathfrak{R} / \Omega$ et $\mathfrak{R}^{\prime} / \Omega$ des extensions de type fini de $\Omega, M$ un modèle de $\Re$ et $M^{\prime}$ un modèle de ${ }^{\prime}{ }^{\prime}$. Nous dirons que $M$ et $M^{\prime}$ sont isomorphes s'il existe un $\Omega$-isomorphisme $J$ de $\mathfrak{R}$ sur $\mathfrak{R}^{\prime}$ tel que les images par $J$ des localités de l'ensemble $M$ soient toutes les localités de l'ensemble $M^{\prime}$; on dit alors aussi que $J$ est un isomorphisme de $M$ sur $M^{\prime}$.

Proposition 3. Tout modèle $M$ d'une extension $\mathfrak{R} / \Omega$ de type fini de $\Omega$ est isomorphe au modèle d'une variété $V$.

Représentons $M$ comme réunion d'un nombre fini de modèles affines $M_{i}(i$ $=1, \ldots, h) ; M_{i}$ est le modèle affine d'un anneau affine $\mathfrak{B}_{i}=\Omega\left[x_{i, 1}, \ldots, x_{i, n(i)}\right]$. Soit $\mathfrak{P}_{i}$ l'idéal premier de l'anneau des polynômes en $n(i)$ lettres à coefficients dans $\Omega$ composé des polynômes $P$ tels que $P\left(x_{i, 1}, \ldots, x_{i, n(i)}\right)=0$. L'ensemble des points $\left(a_{1}, \ldots, a_{n(i)}\right)$ de l'espace affine de dimension $n(i)$ tels que $P\left(a_{1}\right.$, $\left.\ldots, a_{n(i)}\right)=0$ pour tout $P \in \mathfrak{P}_{i}$ est l'ensemble des points d'une variété affine $V_{i}$. Soient $f_{i j}(1 \leqq j \leqq n(i))$ les fonctions coordonnées sur $V_{i}$; il est clair qu'il existe un isomorphisme $J_{i}$ de $\Re\left(V_{i}\right)$ sur $\Re$ qui applique $f_{i j}$ sur $x_{i j}(1 \leqq j \leqq n(i))$ et qui coïncide avec l'identité sur $\Omega$. Cet isomorphisme est un isomorphisme du modèle $M\left(V_{i}\right)$ de $V_{i}$ sur $M_{i}$. Si $i$ et $j$ sont des indices entre 1 et $h, J_{j}^{-1} \circ J_{i}$ est un isomorphisme $T_{j i}^{*}$ de $\mathfrak{R}\left(V_{i}\right)$ sur $\mathfrak{R}\left(V_{j}\right)$. Or, on a le résultat suivant:

Lемме 2. Soient $U$ et $V$ des varietés affines et $T^{*}$ un isomorphisme de $\mathfrak{N}(U)$ sur $\mathfrak{R}(V)$. L'isomorphisme $T^{*}$ peut alors se définir au moyen d'une cor- 
respondance birationnelle $T$ entre $U$ et $V$, qui est uniquement determinée. Si $P \in U$ et $Q \in V$ se correspondent par $T$, une condition nécessaire et suffisante pour que ces points se correspondent régulièrement est que $T^{*}$ applique l'anneau local de $P$ sur celui de $Q$.

Soient $f_{i}(1 \leqq i \leqq m)$ et $g_{j}(1 \leqq j \leqq n)$ les fonctions coordonnées sur $U$ et sur $V$ respectivement. On peut évidemment trouver un corps $K$ qui est un corps de définition pour $U$ et pour $V$ et qui est tel que les $T^{*}\left(f_{i}\right)(1 \leqq i \leqq m)$ appartiennent à $K\left(g_{1}, \ldots, g_{n}\right)$ et les $\left(T^{*}\right)^{-1}\left(g_{j}\right)$ à $K\left(f_{1}, \ldots, f_{m}\right)$. Soit $M$ un point générique de $U$ par rapport à $K$, et soient $x_{1}, \ldots, x_{n}$ les coordonnées de $M$; soit de même $N$ un point générique de $V$ par rapport à $K$, de coordonnées $y_{1}, \ldots, y_{n}$. Il y a un $K$-isomorphisme $\lambda$ de $K(M)$ sur $K\left(f_{1}, \ldots, f_{m}\right)$ tel que $\lambda\left(x_{i}\right)=f_{i}(1 \leqq i \leqq m)$ et un $K$-isomorphisme $\mu$ de $K\left(g_{1}, \ldots, g_{n}\right)$ sur $K(N)$ tel que $\mu\left(g_{j}\right)=f_{j}(1 \leqq j \leqq n)$. Par ailleurs, il est clair que $T^{*}$ induit un $K$-isomorphisme de $K\left(f_{1}, \ldots, f_{m}\right)$ sur $K\left(g_{1}, \ldots, g_{n}\right) ; \mu \circ T^{*} \circ \lambda$ est alors un $K$-isomorphisme de $K(M)$ sur $K(N)$, et définit par suite une correspondance birationnelle $T$ entre $U$ et $V$. Il est clair que $T^{*}$ est l'isomorphisme de $\Re(U)$ sur $\Re(V)$ défini par la correspondance $T$. La connaissance de $T^{*}$ détermine, en vertu de la prop. 1 , les couples de points $(P, Q)$ tels que $P \times Q$ appartienne à $T ; T$ est donc uniquement determinée par $T^{*}$. Supposons maintenant que $P$ et $Q$ se correspondent birégulièrement; choisissons pour point générique $N$ de $V$ le point de $V$ qui correspond à $M$, de sorte que $K(M)=K(N)$; l'anneau de spécialisation de $P$ dans $K(M)$ est alors identique à celui de $Q$ dans $K(N)$ ([W], cor, 3 au th. 17, IV, p. 111). Si $f$ est une fonction appartenant à l'anneau local de $P$, et définie sur $K, f$ est l'image par $\lambda$ d'un élément $u$ de l'anneau de spécialisation de $P$ dans $K(M)$, ̀̀ savoir $u=f(P)$, et on a $\left(T^{*}(f)\right)(Q)=f(P)=u$; puisque $u$ appartient à l'anneau de spécialisation de $Q$ dans $K(N), T^{*}(f)$ appartient à l'anneau local $\mathrm{o}(Q)$ de $Q$. Comme $K$ peut être un sous-corps quelconque de $\Omega$ possédant les propriétés énoncées plus haut, on voit que $T^{*}$ applique $\mathfrak{b}(P)$ dans $\mathfrak{v}(Q)$; on voit de la même manière que $T^{*-1}$ applique $\mathfrak{v}(Q)$ dans $\mathfrak{o}(P)$. Supposons maintenant réciproquement que $T^{*}(\mathfrak{o}(P))=\mathfrak{o}(Q)$. Les $T^{*}\left(f_{i}\right)$ étant dans $\wp(Q)$, les images des $x_{i}$ par l'isomorphisme $\mu \circ T^{*} \circ \lambda$ de $K(M)$ sur $K(N)$ sont dans l'anneau de spécialisation de $Q$ dans $K(N)$; mais, $N$ étant le point qui correspond à $M, \mu \circ T^{*} \circ \lambda$ est l'automorphisme identique, et les $x_{i}$ appartiennent à l'anneau de spécialisation de $Q$ dans $K\left(N^{\top}\right)$. On voit de même que les $y_{j}$ 
appartiennent à l'anneau de spécialisation de $P$ dans $K(M)$; il en résulte immédiatement que $P$ et $Q$ se correspondent régulièrement.

Ceci dit, revenons à la démonstration de la prop. 3. Pour chaque couple $(i, j), T_{j i}^{*}$ définit une correspondance birationnelle $T_{j i}$ entre $V_{i}$ et $V_{j}$, et ces correspondances sont manifestement cohérentes. Montrons qu'elles sont partour birégulières. Soient $P_{i}, P_{j}$ des points de $V_{i}, V_{j}$ tels que $P_{i} \times P_{j}$ appartienne à $T_{j i}$; soient $\mathfrak{o}\left(P_{i}\right)$ et $\mathfrak{o}\left(P_{j}\right)$ leurs anneaux locaux, et $\mathfrak{\downarrow}_{i}, \mathfrak{p}_{j}$ leurs idéaux maximaux. Il résulte alors immédiatement de la prop. 1 que l'idéal engendré par $J_{i}\left(\mathfrak{p}_{i}\right)$ et $J_{j}\left(\mathfrak{p}_{j}\right)$ dans l'anneau engendré par $J_{i}\left(\mathfrak{o}\left(P_{i}\right)\right)$ et $J_{j}\left(\mathfrak{o}\left(P_{j}\right)\right)$ ne contient pas 1 . Mais $J_{i}\left(\mathfrak{D}\left(P_{i}\right)\right)$ et $J_{j}\left(\mathfrak{o}\left(P_{j}\right)\right)$ sont des localités du modèle $M$, et leurs idéaux maximaux sont $J_{i}\left(p_{i}\right)$ et $J_{j}\left(p_{j}\right)$ respectivement. Comme deux localités de $M$ ne peuvent se correspondre sans être identiques, on a $J_{i}\left(\mathfrak{D}\left(P_{i}\right)\right)=J_{j}\left(\mathfrak{D}\left(P_{j}\right)\right)$, doù $T_{j i}^{*}\left(\mathfrak{D}\left(P_{i}\right)\right)$ $=\mathrm{D}\left(P_{j}\right)$. Il en rèsulte que $P_{i}$ et $P_{j}$ se correspondent régulièrement. Posons $F_{i}$ $=\varnothing$ pour tout $i$; alors les variétés affines $V_{i}$, les frontières $F_{i}$ et les correspondances birationnelles $T_{j i}$ définissent une variété $V$. Pour chaque $i$, il y a un isomorphisme $J_{i}^{\prime}$ de $\Re\left(V_{i}\right)$ sur $\Re(V)$ qui applique les $f_{i j}$ sur les fonctions coordonnées sur $V$ relatives à $V_{i}$; il est clair que $T_{j i}^{k}=J_{j}^{\prime-1} \circ J_{l}^{\prime}$. Il y a donc un isomorphisms $H$ de $\Re$ sur $\Re(V)$ tel que l'on ait $J_{i}^{\prime}=H \circ J_{i}$ pour tout $i$; il est clair que $H$ est un isomorphisme du modèle $M$ sur le modèle de $V$.

Remarquons maintenant que le lemme 2 s'étend aux variétés quelconques:

Proposition 4. Soient $U$ et $V$ des variétés et $T^{*}$ un isomorphisme de $\mathfrak{R}(U)$ sur $\Re(V)$. L'isomorphisme $T^{*}$ peut alors se définir au moyen d'une correspondance birationnelle $T$ entre $U$ et $V$, qui est uniquement determinée. Si $P \in U$ et $Q \in V$ se correspondent par $T$, une condition nécessaire et suffisante pour que ces points se correspondent régulièrement est que $T^{*}$ applique l'anneau local de $P$ sur celui de $Q$.

Supposons la variété $U$ donnée par des variétés affines $U_{\alpha}$, par des frontières $F_{\alpha}$ sur les $U_{\alpha}$ et par des correspondances birationnelles $A_{\beta \alpha}$, et $V$ par des variétés affines $V_{\Upsilon}$, des frontières $G_{\Upsilon}$ et des correspondances birationnelles $B_{\delta}$. Nous identifierons comme nous l'avons déjà fait plusieurs fois les $\Re\left(U_{\alpha}\right)$ à $\Re(U)$ et les $\Re\left(V_{\Upsilon}\right)$ à $\mathfrak{R}(V)$; les isomorphismes $A_{\beta \alpha}^{*}$, $B_{\delta \Upsilon}^{*}$ associés aux correspondances $A_{\beta \alpha}, B_{\delta r}$ deviennent alors des automorphismes identiques. Pour chaque couple $(\alpha$, $\gamma), T^{*}$ définit une correspondance birationnelle $T_{\gamma_{\alpha}}$ entre $U_{\alpha}$ et $V_{\gamma}$. Supposons 
qu'un point $P \in U$ ait des représentants $P_{\alpha}$ et $P_{\beta}$ dans $U_{\alpha}$ et $U_{\beta}$ et qu'un point $Q \in V$ ait des représentants $Q_{r}$ et $Q_{\delta}$ dans $V_{r}$ et $V_{\delta}$. Nous allons voir que, si $P_{\alpha} \times Q_{r}$ appartient à $T_{\check{\prime} \alpha}, P_{\beta} \times Q_{\delta}$ appartient à $T_{\delta \beta}$. On a en effet $\mathfrak{D}(P)=\mathfrak{D}\left(P_{\alpha}\right)$ $=\mathfrak{D}\left(P_{\curlyvee}\right), \mathfrak{o}(Q)=\mathfrak{b}\left(Q_{\beta}\right)=\mathfrak{o}\left(Q_{\delta}\right)$; soient $\mathfrak{p}$ et $\mathfrak{q}$ les idéaux maximaux de ces anneaux. Il résulte de l'hypothèse faite que l'idéal engendré par $T^{*}(p)$ et $q$ dans l'anneau engendré par $T^{*}(\mathfrak{o}(P))$ et $\mathfrak{o}(Q)$ ne contient pas 1 ; on en conclut que $P_{\beta} \times Q_{\delta}$ appartient à $T_{\delta \beta}$. Il résulte de là que les $T_{\gamma \alpha}$ sont les représentants dans les $U_{\alpha} \times V_{\curlyvee}$ d'une sous-variété $T$ de $U \times V$; il est clair que $T$ est une correspondance birationnelle et que l'isomorphisme correspondant de $\Re(U)$ sur $\Re(V)$ est $T^{*}$. Le fait que $T$ est uniquement determinée et la deuxième assertion de la prop. 4 résultent immédiatement du lemme 2.

Proposition 5. Soient $U$ et $V$ des variétés. Pour qu'il existe une correspondance birationnelle partout birégulière entre $U$ et $V$, il faut et suffit que les modèles de $U$ et de $V$ soient isomorphes.

Cela résulte immédiatement de la prop. 4.

II.

Nous allons maintenant proposer une nouvelle définition de la notion de variété. Nous désignerons toujours par $\Omega$ un "domaine universel" qui sera considéré comme fixe. Soit $E$ un ensemble. Nous appellerons structure de variété sur $E$ une structure constituée par la donnée d'un ensemble $\Re$ d'applications de parties de $E$ dans $\Omega$ qui possède les propriétés suivantes:

I. Si $f, g \in \Re$, il existe des éléments $u, v$ de $\Re$ qui possèdent les proprietés suivantes: si $P$ est un point de $E$ en lequel $f$ et $g$ sont tous deux definis, $u$ et $v$ sont définis en $P$ et on a $u(P)=f(P)+g(P), v(P)=u(P) g(P)$; de plus, ces éléments $u$ et $v$ sont uniquement determinés. On les désigne par $f+g$ et $f g$.

II. Les lois de composition $(f, g) \rightarrow f+g$ et $(f, g) \rightarrow f g$ définissent sur $\mathfrak{R}$ une structure de corps.

III. Toute application constante de E dans $\Omega$ appartient à $\Re$.

Il est clair que les applications constantes de $E$ dans $\Omega$ forment un souscorps $\Omega^{\prime}$ de $\Re$, isomorphe à $\Omega$, et que nous identifierons à $\Omega$ en identifiant l'application constante de valeur $a$ à l'élément $a$ de $\Omega$.

IV. Si $P \in E$. dósignons par $\mathfrak{o}(P)$ l'ensemble des $f \in \mathfrak{R}$ dont les doinaines de 
définition contiennent $P$; l'application $P \rightarrow \mathfrak{D}(P)$ est alors une application biunivoque de $E$ sur un modèle du corps $\mathfrak{i}$.

(La notion de modèle n'étant définie que pour les extensions de type fini de $\Omega$, IV implique que l'extension $\Re / \Omega$ est de type fini). Nous appellerons désormais "variétés $(W)$ " les variétés au sens défini par A. Weil. Si $V$ est une variété $(W)$, l'ensemble des points de $V$ possède une structure évidente de variété, le corps $\mathfrak{R}$ étant le corps des fonctions sur $V$. De plus, si $U$ et $V$ sont des variétés $(W)$, une condition nécessaire et suffisante pour que les structures de variétés de $U$ et de $V$ soient isomorphes est qu'il existe une correspondance birationnelle partout birégulière entre $U$ et $V$, comme il résulte aussitôt de la prop. 5 , I.

Réciproquement, nous allons voir que toute variété $E$ est isomorphe à une variété portée par une variété $(W)$. Utilisons les mêmes notations que plus haut. Soit $M$ le modèle de $\Re$ formé des anneaux $o(P)$ pour $P \in E$. Nous savons qu'il existe une variété $(W)$, soit $V$, dont le modèle $M(V)$ est isomorphe à $M$; soit $T^{*}$ un isomorphisme de $M(V)$ avec $M$. Soit $E_{r}$ l'ensemble des points de $V$. Il existe donc une application biunivoque $H$ de $E$ sur $E_{\text {- }}$ telle que, pour tout $P \in E, \mathfrak{o}(P)$ soit l'image par $T^{*}$ de l'anneau local $\mathfrak{o}(H(P)) \mathrm{du}$ point $H(P)$ de $E_{i}$. Nous voulons montrer que, si $f \in \mathfrak{g}(H(P))$, on a $f(H(P))$ $=\left(T^{*}(f)\right)(P)$; il en résultera évidemment que $H$ est un isomorphisme de $E_{\mathrm{l}}$, muni de sa structure de variété, sur la variété $E$. Or, il est clair que $f-f(H(P))$ n'est pas inversible dans $\mathfrak{D}(H(P))$; puisque $T^{*}$ laisse les éléments de $\Omega$ fixes, on en conclut que $T^{*}(f)-f(H(P))$ n'est pas inversible dans $\mathrm{o}(P)$. Il en est de même de $T^{*}(f)-\left(T^{*}(f)\right)(P)$; en effet, si cet élément $h$ avait un inverse $g$ dans $\mathfrak{D}(P)$, on aurait, $h(P) g(P)=1$, ce qui est impossible puisque $h(P)=0$. Or $\mathfrak{D}(P)$ est un anneau local; les éléments non inversibles de cet anneau forment donc un idéal, et on en conclut que

$$
\left(T^{*}(f)\right)(P)-f(H(P))=\left(T^{*}(f)-f(H(P))\right)-\left(T^{*}(f)-\left(T^{*}(f)\right)(P)\right)
$$

n'est pas inversible. Mais le premier membre de cette formule représente un élément de $\Omega$, qui ne peut manquer d'être inversible que s'il est nul. Notre assertion est donc établie.

Soit $E$ un ensemble muni d'une structure de variété. Les notations étant les mêmes que plus haut, nous dirons que les éléments de $\mathfrak{R}$ sont les fonctions sur $E$, et que, si $P \in E, \mathfrak{D}(P)$ est l'anneau local de $P$. Il résulte du raisonnement 
que nous venons de faire que l'idéal maximal de l'anneau $\mathfrak{o}(P)$ est l'ensemble des fonctions $f$ de cet anneau telles que $f(P)=0$.

Le corps $\Re$ des fonctions sur $E$ sera désigné par $\mathfrak{R}(E)$, et le modèle de $\Re$ dont les éléments sont les anneaux locaux $\mathfrak{o}(P), P \in E$, par $M(E) ; M(E)$ s'appelle le modèle de $E$. Si $M(E)$ est un modèle affine, on dit que $E$ est une variété affine: $M(E)$ est alors le modèle affine associé à un anneau affine $\mathfrak{B}$ de $\Re(E)$. Cet anneau est uniquement déterminé, comme il résulte du

Lemme 1. Si $\mathfrak{B}$ est un anneau affine de $\mathfrak{N}, \mathfrak{B}$ est l'intersection de tous les anneaux de son modèle affine.

Il est clair que $\mathfrak{B}$ est contenu dans cette intersection $\mathfrak{B}^{\prime}$. Soit réciproquement $x$ un élément de $\mathfrak{F}^{\prime}$. Les éléments $y \in \mathfrak{B}$ tels que $x y \in \mathfrak{B}$ forment évidemment un idéal $\mathfrak{f}$ de $\mathfrak{B}$. Si $\mathfrak{M}$ est un idéal maximal de $\mathfrak{B}, x$ appartient à l'anneau local de $\mathfrak{M}$, d'où il résulte immédiatement que $\mathfrak{\uparrow}$ contient un élément $y$ n'appartenant pas à $\mathfrak{M}$. L'idéal $\mathfrak{f}$, qui n'est contenu dans aucun idéal maximal de $\mathfrak{B}$, doit contenir 1 , d'où $x \in \mathfrak{B}$.

L'anneau affine du modèle d'une variété affine $E$ s'appelle l'anneau affine de $E$.

Soit $E$ une variété. Si $\mathfrak{u}$ est un ensemble quelconque de parties de $E$, nous appellerons topologie engendrée par $\mathfrak{U}$ la topologie la moins fine sur $E$ dans laquelle les ensembles de $\sharp$ sont ouverts; les ensembles ouverts de cette topologie sont ceux du plus petit ensemble de parties de $E$ contenant $\mathfrak{u}$ tel que l'intersection d'un nombre fini $(\neq 0)$ de parties et la réunion d'un nombre quelconque de parties appartenant à cet ensemble appartienne à cet ensemble et que $E$ appartienne à l'ensemble. Si $\mathfrak{B}$ est un anneau affine de $\mathfrak{R}(E)$, nous désignerons

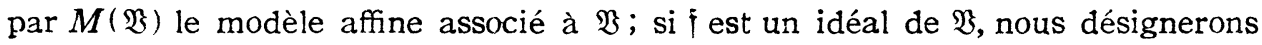
$\operatorname{par} M(\mathfrak{B} ; \mathfrak{f})$ l'ensemble des anneaux locaux de ceux des idéaux maximaux de $\mathfrak{B}$ qui ne contiennent pas $\mathfrak{f}$. Si $M(\mathfrak{B})$ est contenu dans $M(E)$, nous désignerons par $U(\mathfrak{B})$ (respectivement: par $U(\mathfrak{B} ; \mathfrak{\uparrow})$ ) l'ensemble des points $P \in E$ dont les anneaux locaux $\mathfrak{o}(P)$ appartiennent à $M(\mathfrak{B})$ (respectivement: à $M(\mathfrak{B} ; \mathfrak{f})$ ). $\mathrm{Si}$ $f \in \Re(E)$, nous désignerons par $D(f)$ l'ensemble des points de $E$ en lesquels $f$ est définie.

ThÉonème 1. La topologie engendrée par la famille des ensembles $D(f)$, pour $f \in \mathfrak{R}(E)$, est identique à cèlle engendrée par les ensembles $U(\mathfrak{B})$, pour tous les anneaux affines $\mathfrak{B}$ de $\mathfrak{M}(E)$ tels que $M(\mathfrak{B})$ soit contenu dans le modèle $M(E)$ 
de E. Si $\mathfrak{B}$ est l'un de ces anneaux, les parities de $U(\mathfrak{B})$ qui sont ouvertes dans

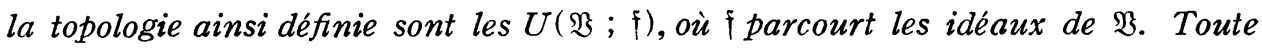
famille non vide d'ensembles ouverts de cette topologie contient un elément maximal. Tout ensemble ouvert peut se représenter comme réunion d'un nombre fini d'ensembles $U(\mathfrak{B})$ relatifs à des anneaux affines $\mathfrak{B}$ tels que $M(\mathfrak{B}) \subset M(E)$.

Soit $\mathfrak{u}$ la famille des réunions finies d'ensembles de la forme $U(\mathfrak{B})$, les $\mathfrak{V}$ étant des anneaux affines tels que $M(\mathfrak{B}) \subset M(E)$.

1. Si $\mathfrak{B}$ est un anneau affine tel que $M(\mathfrak{B}) \subset M(E), U(\mathfrak{B})$ se compose des points $P \in E$ dont les anneaux locaux contiennent $\mathfrak{B}$. Il est en effet clair que $\mathfrak{B} \subset \mathfrak{n}(P)$ pour tout $P \in U(\mathfrak{B})$. Soit réciproquement $P$ un point de $E$ tel que $\mathfrak{B} \subset \mathfrak{D}(P)$. Soient $\mathfrak{p}$ l'idéal maximal de $\mathfrak{o}(P)$ et $\mathfrak{M}$ l'idéal $\mathfrak{p} \cap \mathfrak{B}$ de $\mathfrak{B} ; \mathfrak{M}$ est donc le noyau de la restriction à $\mathfrak{V}$ de l'homomorphisme $f \rightarrow f(P)$ de $\mathfrak{D}(P)$ sur $\Omega$, ce qui montre que $\mathfrak{M}$ est un idéal maximal de $\mathfrak{B}$; son anneau local peut donc se mettre sous la forme $\mathfrak{o}\left(P^{\prime}\right)$, avec un $P^{\prime} \in E$. Un élément de $\mathfrak{B}$ non situé dans $\mathfrak{M}$ étant inversible dans $\mathfrak{o}(P)$, on a $\mathfrak{o}\left(P^{\prime}\right) \subset \mathfrak{o}(P)$; l'idéal maximal de $\mathfrak{n}\left(P^{\prime}\right)$, qui est engendré par $\mathfrak{M}$, étant contenu dans $\mathfrak{p}$, il est clair que $\mathfrak{D}(P)$ et $\mathfrak{n}\left(P^{\prime}\right)$ se correspondent, d'où $\mathfrak{o}(P)=\mathfrak{o}\left(P^{\prime}\right)$ et $P \in U(\mathfrak{B})$. Soient $f_{1}, \ldots, f_{m}$ des éléments de $\mathfrak{B}$ tels que $\mathfrak{B}=\Omega\left[f_{1}, \ldots, f_{m}\right]$; pour que $\mathfrak{B} \subset_{\mathfrak{D}}(P)$, il faut et suffit que l'on ait $f_{i} \in \mathfrak{o}(P)$, i.e. $P \in D\left(f_{i}\right)(1 \leqq i \leqq m)$; on a donc $U(\mathfrak{B})=D\left(f_{1}\right) \cap \ldots$ $\cap D\left(f_{m}\right)$.

2. L'anneau $\mathfrak{B}$ étant comme dans 1 , et $f$ une fonction sur $E$, il y a un idéal $\mathfrak{f} \neq\{0\}$ de $\mathfrak{B}$ tel que $D(f) \cap U(\mathfrak{B})=U(\mathfrak{B} ; \mathfrak{\mathfrak { f }})$. Soit en effet $\mathfrak{f}$ l'ensemble des $x \in \mathfrak{B}$ tels que $x f \in \mathfrak{B}$; c'est évidemment un idéal $\neq\{0\}$. Soit $P$ un point de $U(\mathfrak{B}) ; \mathfrak{D}(P)$ est alors l'anneau local d'un idéal maximal $\mathfrak{M}$ de $\mathfrak{B}$. Pour que $P \in D(f)$, il faut et suffit que $f \in \mathfrak{D}(P)$, donc qu'il y ait un $x \in \mathfrak{B}$ n'appartenant pas à $\mathfrak{M}$ tel que $x f \in \mathfrak{B}$ : cela signifie que $\mathfrak{M}$ ne doit pas contenir $\mathfrak{f}$. L'anneau $\mathfrak{B}$ étant affine, donc isomorphe à un anneau quotient d'un anneau de polynômes, il est bien connu que, pour tout $x \neq 0$ de $\mathfrak{B}$, il y a un idéal maximal $\mathfrak{M}$ de $\mathfrak{F}$ ne contenant pas $x$; on en conclut que $D(f) \cap U(\mathfrak{B}) \neq \varnothing$.

3. L'anneau $\mathfrak{B}$ étant comme dans 1 , et $\mathfrak{\uparrow}$ étant un idéal $\neq\{0\}$ de $V, U(\mathfrak{B} ; \mathfrak{f})$ appartient à $\mathfrak{u}$. Cela résulte du lemme $1, \mathrm{I}$.

4. Si $f_{1}, \ldots, f_{m}$ sont dans $\Re(E), D\left(f_{1}\right) \cap \ldots \cap D\left(f_{m}\right)$ est un ensemble non vide de $\mathfrak{u}$. Pour $m=1$, cela résulte de ce que $E \in \mathfrak{u}$ (en vertu de la définition d'un modèle) et de ce que, si $\mathfrak{B}$ est un anneau affine de $\mathfrak{R}(E)$ tel que 
$M(\mathfrak{B}) \subset M(E), U(\mathfrak{B}) \cap D(f)$ est un ensemble non vide de $\mathfrak{l}$ en vertu de 2 . et 3. Dans le cas général, on procède par récurrence sur $m$. Si $m>1$ et si notre assertion est vraie pour $m-1, D\left(f_{1}\right) \cap \ldots \cap D\left(f_{m-1}\right)$ est non vide et est la réunion d'un nombre fini d'ensembles de la forme $U(\mathfrak{V})$, les $\mathfrak{B}$ étant des anneaux affines tels que $M(\mathfrak{B}) \subset M(E)$, et l'intersection de chacun de ces ensembles avec $D\left(f_{m}\right)$ est dans $\mathfrak{H}$.

Il résulte déjà de 1 . et de 4 . que l'intersection de deux ensembles de $\mathfrak{l}$ est dans $\mathfrak{U}$ et que les topologies engendrées par les ensembles $U(\mathfrak{B})$, où les $\mathfrak{B}$ parcourent les anneaux affines de $\mathfrak{N}(E)$ tels que $M(\mathfrak{B}) \subset M(E)$, et par les $D(f)$, $f \in \Re(E)$, sont identiques.

5. Soit $\mathfrak{B}$ comme dans 1 , et soient $\mathfrak{f}, \mathfrak{f}^{\prime}$ des idéaux de $\mathfrak{B} ;$ on a alors $U(\mathfrak{B} ; \mathfrak{f}) \cup U\left(\mathfrak{B} ; \mathfrak{f}^{\prime}\right)=U\left(\mathfrak{B} ; \mathfrak{\mathfrak { f }}+\mathfrak{f}^{\prime}\right), \quad U(\mathfrak{B} ; \mathfrak{f}) \cap U\left(\mathfrak{B} ; \mathfrak{f}^{\prime}\right)=U\left(\mathfrak{B} ; \mathfrak{f f}^{\prime}\right)$. En effet, pour qu'un idéal premier de $\mathfrak{B}$ ne contienne pas $\mathfrak{f}+\mathfrak{f}^{\prime}$, il faut et suffit qu'il ne contienne pas à la fois $\mathfrak{f}$ et $\mathfrak{f}^{\prime}$, et, pour qu'il ne contienne pas $\mathrm{ff}^{\prime}$, il faut et suffit qu'il ne contienne ni $\mathfrak{f}$ ni $\mathfrak{f}^{\prime}$. Il résulte immédiatement de là que, pour tout $U \in \mathfrak{U}, U \cap U(\mathfrak{B})$ est de la forme $U(\mathfrak{B} ; \mathfrak{f})$ pour un idéal $\mathfrak{f}$ convenable. Réciproquement, tout ensemble de cette forme est dans $\mathfrak{U}$ (lemme $1, I$ ).

6. L'anneau $\mathfrak{B}$ étant comme dans 1 , et $U$ un ensemble de $\mathfrak{u}$ contenu dans $U(\mathfrak{B})$, il y a en général plusieurs idéaux $\mathfrak{f}$ tels que $U=U(\mathfrak{B} ; \mathfrak{\uparrow})$; mais, parmi eux, il y en a un qui contient tous les autres, à savoir l'intersection $f(U)$ de tous les idéaux maximaux dont les anneaux locaux sont les anneaux locaux de points de $U(\mathfrak{B})$ n'appartenant pas à $U$. En effet, il est clair que si un idéal maximal $\mathfrak{M}$ ne contient pas $\mathfrak{f}(U)$, son anneau local est l'anneau local d'un point de $U$. Par ailleurs, si $U=U(\mathfrak{B} ; \mathfrak{f})$, il est clair que $\mathfrak{f} \subset \mathfrak{f}(U)$, et, si un idéal maximal ne contient pas $\mathfrak{f}$, il ne contient pas non plus $\mathfrak{f}(U)$. Si $U, U^{\prime}$ sont des ensembles de $\mathfrak{u}$ contenus dans $U(\mathfrak{B})$, une condition nécessaire et suffisante pour que $U \subset U^{\prime}$ est que $\mathfrak{f}(U) \subset \mathfrak{f}\left(U^{\prime}\right)$. Or, l'anneau $\mathfrak{B}$ étant noethérien, tout ensemble non vide d'idéaux de $\mathfrak{B}$ admet un élément maximal. Il en résulte que toute famille non vide de parties de $U$ appartenant à $\mathfrak{u}$ contient un élément maximal.

7. Soit $\left(U_{n}\right)$ une suite croissante d'ensembles appartenant à $\mathfrak{H}$. Mettons $E$ sous la forme $U\left(\mathfrak{B}_{1}\right) \cup \ldots \cup U\left(\mathfrak{B}_{h}\right)$, où les $\mathfrak{B}_{i}$ sont des anneaux affines de $\mathfrak{N}(E)$ tels que les $M\left(\mathfrak{B}_{i}\right)$ soient contenus dans $M(E)$. Pour chaque $i$, les membres de la suite $\left(U_{n} \cap U\left(\mathfrak{B}_{i}\right)\right)$ sont tous égaux à partir d'un certain rang; il en ré- 
sulte immédiatement que les $U_{n}$ sont tous égaux à partir d'un certain rang. Ceci montre que toute partie non vide de $\mathfrak{u}$ admet un élément maximal. Il en résulte que toute réunion (finie ou infinie) d'ensembles $U_{\alpha}$ appartenant à $\mathfrak{l}$ appartient à $\mathfrak{U}$ (il suffit d'appliquer le résultat précédent à la famille des réunions finies d'ensembles $U_{\alpha}$ ). On en conclut immédiatement que $\mathfrak{u}$ est la famille de tous les ouverts de la topologie considérée. Le théorème 1 est donc démontré.

La topologie définie dans l'énonce du théorème 1 s'appelle la topologie de Zariski sur $E$; elle n'est en général pas séparée. D’une manière générale, nous appellerons espace de Zariski un espace topologique dans lequel toute famille non vide d'ensembles ouverts admet un élément maximal, c'est-à-dire aussi dans lequel toute famille non vide d'ensembles fermés admet un élément minimal. Il est clair que tout sous-espace d'un espace de Zariski est un espace de Zariski.

Soit $E$ un espace de Zariski. Une partie $A$ de $E$ est dite irréductible si les conditions suivantes sont satisfaites: a) $A$ n'est pas vide; b) il n'existe aucune représentation de $A$ comme réunion de deux parties relativement fermées de $A$ toutes deux distinctes de $A$. Pour qu'il en soit ainsi, il faut et suffit que l'adhérence $\bar{A}$ de $A$ soit irréductible. Car, supposons $A$ irréductible, et soit $\bar{A}=$ $B \cup B^{\prime}$, où $B, B^{\prime}$ sont fermés; alors $A=(B \cap A) \cup\left(B^{\prime} \cap A\right)$, et, comme $B \cap A$, $B^{\prime} \cap A$ sont relativement fermés, l'un de ces ensembles, soit $B \cap A$, est $A$ tout entier ; comme $B$ est fermé, $B=\bar{A}$, et $\bar{A}$ est irréductible. Supposons réciproquement $\bar{A}$ irréductible. Toute partie relativement fermée de $A$ est l'intersection de $A$ avec une partie fermée de $\bar{A}$; soient $B, B^{\prime}$ des parties fermées de $\bar{A}$ telles que $A=(B \cap A) \cup\left(B^{\prime} \cap A\right)$. Comme $B \cup B^{\prime}$ est fermé et contient $A$, on a $B \cup B^{\prime}=\bar{A}$, et l'un des ensembles $B, B^{\prime}$, soit $B$, es̀t $\bar{A}$ tout entier, d'où $B \cap A=A$, de sorte que $A$ est irréductible. Il résulte immédiatement de la définition que, pour qu'une partie non vide $A$ de $E$ soit irréductible, il faut et suffit que lintersection de deux parties relativement ouvertes non vides de $A$ soit toujours non vide, ou encore que tout ensemble relativement ouvert non vide de $A$ soit dense dans $A$; ceci entraine que l'intersection d'un nombre fini quelconque de parties relativement ouvertes non vides de $A$ est non vide et dense dans $A$. De plus, il résulte de la partie 4 de la démonstration du théoréme 1 que, si $E$ est une variété, $E$ est un ensemble irréductible.

ThÉorème 2. Soit E un espace de Zariski. Toute partie A de E peut alors 
se représenter comme réunion d'un nombre fini de parties relativement fermées irréductibles $A_{1}, \ldots, A_{h}$ de $A$ telles que $A_{i}$ ne soit pas contenu dans $A_{j}$ si $i \neq j$; les $A_{i}$ sont uniquement déterminés et toute partie irréductible de A est contenue dans l'un de ces ensembles.

Soit $\mathfrak{Z}$ l'ensemble des réunions finies de parties irréductibles de $E$. Montrons que toute partie fermée de $E$ est dans $\mathfrak{A}$. Supposons le contraire; l'ensemble des parties fermées de $E$ n'appartenant pas à $\mathfrak{U}$ admet alors un élément minimal $B$. Il est clair que $B$ n'est ni vide ni irréductible. On a donc $B=$ $B^{\prime} \cup B^{\prime \prime}$, où $B^{\prime}$ et $B^{\prime \prime}$ sont fermés et distincts de $B$. Il résulte du caractère minimal de $B$ que $B^{\prime}$ et $B^{\prime \prime}$ sont dans $\mathfrak{U}$; la réunion de deux ensembles de $\mathfrak{A}$ étant évidemment dans $\mathfrak{A}$, on a $B \in \mathfrak{A}$, d'où contradiction. Soit $A$ une partie quelconque de $E$ : son adhérence $\bar{A}$ est alors dans $\mathfrak{A}$, et peut par suite se représenter comme réunion d'ensembles irréductibles $B_{1}, \ldots, B_{h}$ dont on peut évidemment supposer qu’ils sont fermés et que $B_{i} \notin B_{j}$ si $i \neq j ; A$ est la réunion des ensembles $A_{i}=A \cap B_{i}$. Montrons que $A_{i}$ est dense dans $B_{i}$. Si $i \neq j$, on a $B_{i} \cap$ $B_{j} \neq B_{i} ; B_{i}$, étant irréductible, n'est donc pas la réunion des $B_{j} \cap B_{i}$ pour tous les $j \neq i$. Mais on a $\bar{A}_{i} \subset B_{i}$ et $\bar{A}$ est la réunion des $\bar{A}_{i} ; B_{i}$ est donc la réunion de $\bar{A}_{i}$ et des $B_{j} \cap B_{i}$ pour $j \neq i ; B_{i}$ étant irréductible, on a $B_{i}=\bar{A}_{i}$. On en conclut que les $A_{i}$ sont irréductibles et que $A_{j} \nsubseteq A_{i}$ si $j \neq i$. Si $A^{\prime}$ est une partie irréductibie de $A, A^{\prime}$ est la réunion des $A^{\prime} \cap A_{i}$ qui sont relativement fermés dans $A^{\prime}$; on a donc $A^{\prime}=A^{\prime} \cap A_{i}, A^{\prime} \subset A_{i}$ pour au moins un $i$. Les $A_{i}$ sont donc toutes les parties irréductibles maximales de $A$; ils sont uniquement déterminés.

Les ensembles $A_{i}$ s'appellent les composantes irréductibles de l'ensemble $A$.

Soit maintenant $A$ une partie irréductible de $E$. On dit qu'une fonction $f \in \mathfrak{R}(E)$ admet une trace sur $A$ si $f$ est défini en au moins un point de $A$. Dans ce cas, on désigne par $\rho_{A}(f)$ la restriction de $f$ à la partie $A \cap D(f)$ de son domaine de définition. Soient $f, f_{1}, \ldots, f_{m}$ des fonctions qui admettent des traces sur $A$; si on a $f(P)=0$ en tout point $P \in A$ en lequel $f, f_{1}, \ldots, f_{m}$ sont toutes définies, on a $\rho_{A}(f)=0$, (i.e. $f(P)=0$ pour tout $P \in A \cap D(f)$ ). Supposons en effet $f \neq 0$; si $\rho_{A}(f)$ était $\neq 0, f^{-1}$ aurait une trace sur $A$ et les ensemble relativement ouverts $D(f) \cap A, D\left(f^{-1}\right) \cap A, D\left(f_{i}\right) \cap A(1 \leqq i \leqq m)$, tous non vides, auraient un point commun $P$; on aurait $f(P)=0$, ce qui est impossible puisque $P \in D\left(f^{-1}\right)$. Appliquant ceci au cas où $f=1$, on voit que des 
fonctions en nombre fini qui ont toutes des traces sur $A$ sont toutes définies simultanément en au moins un point de $A$. Il en résulte immédiatement que l'ensemble $\mathfrak{o}(A)$ des fonctions qui ont des traces sur $A$ est un sous-anneau de $\mathfrak{R}(E)$, contenant évidemment $\Omega$. Soit $\mathfrak{p}$ l'ensemble des $f \in \mathfrak{D}(A)$ tels que $\rho_{A}(f)$ $=0 ;$ si $f, g$ sont dans $\mathfrak{p}$ et $h$ dans $\mathfrak{b}(A), f-g$ et $f \hat{h}$ prennent la valeur 0 en tout point où $f, g, h$ sont définies, d'où $f-g \in \mathfrak{p}, f h \in \mathfrak{p} ; \mathfrak{p}$ est donc un idéal de $n(A)$. Si $f$ est un élément de $\mathfrak{D}(A)$ non situé dans $\mathfrak{p}$, il y a un $P \in A$ tel que $P \in D(f)$, $f(P) \neq 0$, d'où $f^{-1} \in \mathfrak{p}(A)$. On en conclut que $\mathfrak{p}$ est l'unique idéal maximal de $\mathfrak{D}(A)$, donc que $\mathfrak{D}(A) / \mathfrak{p}$ est un corps. Si $f^{*}$ est un élément quelconque de ce corps, nous désignerons par $D\left(f^{*}\right)$ la réunion des ensembles $A \cap D(f)$ pour tous les $f \in \mathfrak{o}(A)$ appartenant à la classe $f^{*}$ modulo $\mathfrak{p}$. Soit $P$ un point de cet ensemble; si $f, g$ sont des représentants de $f^{*}$ tous deux définis en $P$, on a $f(P)=g(P)$ puisque $f-g \in \mathfrak{p}$; nous désignerons par $\sigma_{A}\left(f^{*}\right)$ la fonction définie sur $D\left(f^{*}\right)$ dont la valeur en un point $P \in D\left(f^{*}\right)$ est la valeur commune en $P$ de tous les $f \in \mathfrak{o}(A)$ qui appartiennent à la classe $f^{*}$ et qui sont définis en $P$. Si $f \in f^{*}$, nous dirons que $\sigma_{A}\left(f^{*}\right)$ est la trace de $f$ sur $A$; cette fonction prolonge évidemment $\rho_{A}(f)$, sans lui être en général identique. Nous désignerons par $\Re(A)$ l'ensemble des traces de fonctions de $\mathfrak{o}(A)$. Si $f^{*}, g^{*}$ sont dans $\mathfrak{o}(A) / \mathfrak{p}$, il y a des éléments uniquement déterminés $u^{*}, v^{*}$ de $\mathfrak{D}(A) / \mathfrak{p}$ tels que $\left(\sigma_{A}\left(f^{*}\right)\right)(P)+\left(\sigma_{A}\left(g^{*}\right)\right)(P)=\left(\sigma_{A}\left(u^{*}\right)\right)(P)$ et $\left(\sigma_{A}\left(f^{*}\right)\right)(P)\left(\sigma_{A}\left(g^{*}\right)\right)(P)=\left(\sigma_{A}\left(v^{*}\right)\right)$ $(P)$ en tout point $P \in D\left(f^{*}\right) \cap D\left(g^{*}\right)$. En effet, $u_{0}^{*}=f^{*}+g^{*}$ et $v_{0}^{*}=f^{*} g^{*}$ possèdent évidemment la propriété requise. Par ailleurs, supposons que $u^{*}, v^{*}$ la possèdent. Soient $f, g, u, v$ des représentants de $f^{*}, g^{*}, u^{*}, v^{*}$; on a donc $u(P)=f(P)+g(P), v(P)=f(P) g(P)$ en tout point $P \in A$ en lequel $f, g, u$, $v$ sont toutes définies, d'où $u-(f+g) \in \mathfrak{p}, v-f g \in \mathfrak{p}$ et $u^{*}=f^{*}+g^{*}, v^{*}=f^{*} g^{*}$. En particulier, l'application $f^{*} \rightarrow \sigma_{A}\left(f^{*}\right)$ de $\mathfrak{D}(A) / \mathfrak{p}$ sur $\Re(A)$ est biunivoque; elle transporte à $\mathfrak{R}(A)$ la structure de corps de $\mathfrak{o}(A) / \mathfrak{p}$. Le corps ainsi défini s'appelle le corps des fonctions sur $A$; il contient toutes les applications constantes de $A$ dans $\Omega$, que nous identifierons aux éléments de $\Omega$.

Nous nous proposons maintenant de déterminer à quelle condition la donnée du corps $\Re(A)$ définit sur $A$ une structure de variété. Les conditions I, II, III du début de II. sont évidemment satisfaites pour $A$ et $\Re(A)$; il reste à examiner la condition IV. Si $P \in A$, nous désignerons par $n_{A}(P)$ l'ensemble des fonctions de $\Re(A)$ qui sont définies en $P$. Nous désignerons d'autre part par $\theta$ 
l'homomorphisme de $\mathfrak{D}(A)$ sur $\mathfrak{R}(A)$ qui applique tout $f \in \mathfrak{D}(A)$ sur sa trace sur $A$. Soient $P$ un point de $A$ et $\mathrm{m}$ l'idéal maximal de $\mathfrak{D}(P)$; on a évidemment $\mathfrak{b}(P) \subset \mathfrak{D}(A), \mathfrak{b} \subset \mathrm{m}$. Montrons que $\theta(\mathfrak{D}(P))=\mathfrak{D}_{A}(P)$, et que $\theta(\mathrm{m})$ est l'idéal des fonctions de $\mathrm{D}_{A}(P)$ qui sont nulles en $P$. Il est clair que la trace sur $A$ de toute fonction $f \in \mathfrak{o}(P)$ est dans $\mathfrak{o}_{A}(P)$ et que, si $f \in \mathrm{m}$, sa trace est nulle en $P$. Réciproquement, si $g \in \mathfrak{D}_{A}(P), g$ est l'image par $\sigma_{A}$ d'un élément $f^{*} \in \mathfrak{D}(A) / \mathfrak{p}$ qui est représenté par un élément $f \in \mathfrak{o}(A)$ défini en $P$, donc appartenant à $\mathfrak{D}(P)$, et, si $g(P)=0$, on a $f(P)=0$, d'où $f \in \mathfrak{m}$; nos assertions sont donc établies. Il en résulte immédiatement que $\theta(\mathrm{m})$ est l'unique idéal maximal de $\mathfrak{o}_{A}(P)$ et que $\mathrm{D}_{A}(P) / \theta(\mathrm{m})$ est isomorphe à $\Omega$. Soient par ailleurs $P^{\prime}$ un point $\neq P$ appartenant à $A$ et $\mathrm{m}^{\prime}$ l'idéal maximal de $\mathfrak{o}\left(P^{\prime}\right)$. L'idéal engendré par $\mathfrak{n}$ ' et $\mathfrak{m}^{\prime}$ dans l'anneau engendré par $\mathfrak{D}(P)$ et $\mathfrak{D}\left(P^{\prime}\right)$ contient 1 ; il en résulte immédiatement que l'idéal engendré par les idéaux maximaux de $\mathfrak{o}_{A}(P)$ et de $\mathfrak{o}_{A}\left(P^{\prime}\right)$ dans l'anneau engendré par ces deux anneaux contient 1 .

Soit $\mathfrak{B}$ un anneau affine de $\mathfrak{R}(E)$ tel que $M(\mathfrak{B}) \subset M(E)$; si $U(\mathfrak{B})$ rencontre $A$, il est clair que $\mathfrak{B} C_{D}(A)$; soit alors $\mathfrak{B}^{A}=\theta(\mathfrak{B})$; c'est un sous-anneau de $\Re(A)$ contenant $\Omega$ et engendré par un nombre fini d'éléments sur $\Omega$. Si $P$ $\in U(\mathfrak{B}) \cap A, \mathfrak{o}(P)$ est l'anneau local d'un idéal maximal $\mathfrak{P}$ de $\mathfrak{B}$, intersection de $\mathfrak{B}$ avec l'idéal maximal $\mathrm{m}$ de $\mathfrak{o}(P)$; il est clair que $\mathfrak{p} \cap \mathfrak{B} \subset \mathfrak{M}$. On a $\mathfrak{o}_{A}(P)$ $=\theta(\mathfrak{o}(P))$, et on vérifie immédiatement que cet anneau est lanneau local de l'idéal premier $\theta(\mathfrak{M})$ de $\mathfrak{B}^{A}$. L'anneau $\mathfrak{B}^{A} / \theta(\mathfrak{M})$ est un sous-anneau contenant $\Omega$ de $\mathfrak{o}_{\Delta}(P) / \theta(\mathfrak{m})=\Omega$; cet anneau est donc $\Omega$, et $\theta(\mathfrak{M})$ est un idéal maximal de $\mathfrak{B}^{A}$. Soit réciproquement $P$ un point de $A$ tel que $\mathfrak{B}^{A} \subset_{\mathfrak{D}_{A}}(P)$; l'intersection $\mathfrak{M}^{A}$ de $\mathfrak{B}^{A}$ avec l'idéal maximal $\mathfrak{m}_{A}$ de $\mathfrak{D}_{A}(P)$ est alors un idéal premier de $\mathfrak{B}^{A}$, et se met sous la forme $\theta(\mathfrak{M})$ où $\mathfrak{M}$ est un idéal de $\mathfrak{B}$ contenant $\mathfrak{p} \cap \mathfrak{B} ; \theta$ définit un isomorphisme de $\mathfrak{B} / \mathfrak{R}$ sur le sous-anneau $\mathfrak{B}^{A} / \mathfrak{M}^{A}$ de $\mathfrak{o}_{A}(P) / \mathrm{m}_{A}=\Omega$; comme $\Omega$ $\subset \mathfrak{B} / \mathfrak{M}$, on a $\mathfrak{B} / \mathfrak{M}=\Omega$ et $\mathfrak{M}$ est un idéal maximal de $\mathfrak{B}$. Puisque $M(\mathfrak{B}) \subset M(E)$, l'anneau local de $\mathfrak{M}$ peut se mettre sous la forme $\mathfrak{D}\left(P^{\prime}\right), P^{\prime}$ étant un point de $E$. Nous allons montrer que $P^{\prime}=P$. L'idéal $\mathfrak{M}$ contient $\mathfrak{p} \cap \mathfrak{B}$, d'où il résulte que $\mathfrak{o}\left(P^{\prime}\right) \subset \mathfrak{D}(A)$; il est clair que $\theta$ applique $\mathfrak{o}\left(P^{\prime}\right)$ sur $\mathrm{o}_{A}(P)$ et lidéal maximal $\mathrm{m}^{\prime}$ de $\mathfrak{o}\left(P^{\prime}\right)$ sur $\mathrm{m}_{A}$. Mais nous savons que l'on a aussi $\theta(\mathfrak{o}(P))=\mathfrak{o}_{A}(P), \theta(\mathrm{m})$ $=\mathrm{m}_{A}$, si $\mathrm{m}$ est l'idéal maximal de $\mathfrak{D}(P)$. L'homomorphisme $\theta$ applique donc l'anneau engendré par $\mathfrak{D}(P)$ et $\mathfrak{D}\left(P^{\prime}\right)$ sur $\mathrm{D}_{A}(P)$ et l'idéal de cet anneau engendré par $\mathfrak{m}$ et $\mathfrak{m}^{\prime}$ sur $\mathfrak{m}_{A}$; ce dernier idéal ne peut donc contenir 1 , d'où $P=P^{\prime}$. 
L'ensemble $U(\mathfrak{B}) \cap A$ est donc l'ensemble des points $P \in A$ tels que $\mathfrak{B}^{A} \subset \mathfrak{D}_{A}(P)$, et, pour tout point $P$ de cet ensemble, $\mathfrak{o}_{A}(P)$ est l'anneau local d'un idéal maximal de $\mathfrak{B}^{A}$. Soit $f$ un élément quelconque de $\mathfrak{D}(A)$; l'ensemble $D(f) \cap \dot{A}$ est donc non vide et relativement ouvert dans $A$; il en est de même de $U(\mathfrak{B}) \cap A$; $A$ étant irréductible, l'ensemble $A \cap D(f) \cap U(\mathfrak{B})$ contient au moins un point $P$; on a $\theta(f) \in \mathfrak{o}_{A}(P)$, et $\mathfrak{o}_{A}(P)$ est l'anneau local d'un idéal maximal de $\mathfrak{B}^{A}$; il en résulte que $f$ appartient au corps des quotients de $\mathfrak{B}^{A}$. Ce dernier est donc $\Re(A)$ tout entier. On en conclut que l'extension $\Re(A) / \Omega$ est de type fini et que $\mathfrak{B}^{A}$ en est un anneau affine. Les $\mathfrak{D}_{A}(P)$ sont donc des localités de $\Re(A)$, et deux distinctes de ces localités ne se correspondent jamais, comme nous l'avons établi plus haut. Si nous désignons par $U_{A}\left(\mathfrak{B}^{A}\right)$ l'ensemble des $P \in A$ tels que $\mathfrak{o}_{A}(P)$ contienne $\mathfrak{B}^{A}$, on a $U_{A}\left(\mathfrak{B}^{A}\right)=U(\mathfrak{B}) \cap A$. Il est clair qu'on peut trouver un nombre fini d'anneaux affines $\mathfrak{B}_{i}(1 \leqq i \leqq h)$ de $\mathfrak{R}(E)$ dont les modèles sont contenus dans $M(E)$, tels que $U\left(\mathfrak{B}_{i}\right) \cap A \neq \varnothing(1 \leqq i \leqq h)$ et que $A$ soit la réunion des $U\left(\mathfrak{B}_{i}\right) \cap A ; A$ est alors la réunion des $U_{A}\left(\mathfrak{B}_{i}^{A}\right)$. Mais $U_{A}\left(\mathfrak{B}_{i}^{A}\right)$ n'est en général pas l'ensemble des anneaux locaux de tous les idéaux maximaux de $\mathfrak{B}_{i}^{A}$.

Soit $B$ l'adhérence de $A$; c'est encore un ensemble irréductible. Si $f \in \mathfrak{o}(B)$, l'ensemble $D(f) \cap B$, non vide et relativement ouvert dans $B$, rencontre $A$; il en résulte immédiatement que $\mathfrak{o}(B)=\mathfrak{o}(A)$ et que $\mathfrak{p}$ est aussi l'ensemble des fonctions de trace nulle sur $B$. Si $f \in \mathrm{o}(A)=\mathrm{n}(B)$, soit $\theta_{B}(f)$ sa trace sur $B$ il est clair que $\theta_{B}(f)$ prolonge la fonction $\theta(f)$ et qu'il y a un isomorphisme $J$ de $\Re(A)$ sur $\Re(B)$ tel que $J(\theta(f))=\theta_{B}(f)$ pour tout $f \in \mathfrak{D}(A)$. L'anneau $\mathfrak{B}$ étant comme plus haut, on peut maintenant affirmer que l'anneau local de tout idéal maximal de $\mathfrak{B}^{A}$ est de la forme $\mathfrak{o}_{B}(P)$, pour un $P \in B$. Il suffit pour cela de montrer que, si $\mathfrak{M}$ est un idéal maximal de $\mathfrak{B}$ contenant $\mathfrak{p} \cap \mathfrak{B}$, et $P$ le point de $E$ tel que $\mathfrak{D}(P)$ soit l'anneau local de $\mathfrak{M}$, on a $P \in B$. Or, le complémentaire $U$ de $U(\mathfrak{B}) \cap B$ par rapport à $U(\mathfrak{B})$ est ouvert; si donc $\mathfrak{f}$ est l'intersection des idéaux maximaux $\mathfrak{N}$ de $\mathfrak{B}$ tels que l'anneau local de $\mathfrak{R}$ soit anneau local d'un point de $U(\mathfrak{B}) \cap B$, l'anneau local de tout idéal maximal de $\mathfrak{B}$ contenant $\mathfrak{f}$ est anneau local d'un point de $U(\mathfrak{B}) \cap B$. Or il est clair que $\mathfrak{p} \cap \mathfrak{B} \subset \mathfrak{f}$ (on voit d'ailleurs facilement que $\mathfrak{f}=\mathfrak{p} \cap \mathfrak{B}$ ), et notre assertion est démontrée. Le corps $\Re(B)$ définit donc sur $B$ une structure de variété. Montrons que la topologie de Zariski de cette variété est celle induite par la topologie de Zariski de $E$. 
Si $\mathfrak{B}$ est un anneau affine de $\Re(E)$ tel que $M(\mathfrak{Z})$ soit contenu dans $M(E)$ et que $U(\mathfrak{B}) \cap B \neq \varnothing$, on a $U(\mathfrak{B}) \cap B=U_{B}\left(\mathfrak{V}^{B}\right)$; il en résulte immédiatement que l'intersection avec $B$ de toute partie ouverte de $E$ est ouverte dans la topologie de Zariski de $B$. Soit maintenant $g$ un élément quelconque de $\mathfrak{R}(B)$; l'ensemble $D_{B}(g)$ des points de $B$ en lesquels $g$ est définie est alors la réunion des $D(f) \cap B$ pour tous les $f \in \mathfrak{o}(B)$ dont la trace sur $B$ est $g$; c'est donc l'intersection de $B$ avec une partie ouverte de $E$, et il en résulte que toute partie ouverte de $B$ (au sens de la topologie de Zariski de $B$ ) est l'intersection avec $B$ d'une partie ouverte de $E$, ce qui démontre notre assertion.

Ceci dit, supposons que la donnée de $\Re(A)$ définisse sur $A$ une structure de variété. L'ensemble des $\cap_{A}(P)$ pour $P \in A$ est alors un modèle de $\mathfrak{R}(A)$. Tenant compte de l'isomorphisme $J$ de $\Re(A)$ sur $\Re(B)$ et observant que l'on a évidemment $J\left(\mathfrak{o}_{A}(P)\right)=\mathfrak{o}_{B}(P)$ si $P \in A$, on voit que l'ensemble des $\mathfrak{n}_{B}(P)$, pour $P \in A$, est un modèle de $\Re(B)$; cela signifie que $A$ est une partie ouverte de la variété $B$. Réciproquement, le même raisonnement, pris dans l'autre sens, montre que, si $A$ est ouvert dans $B$, la donnée de $\Re(A)$ définit sur $A$ une structure de variété.

Lorsque l'ensemble irréductible $A$ est ouvert dans son adhérence, nous dirons que $A$, muni de la structure de variété définie par $\Re(A)$, est une sousvariété de $E$. Nous nous écartons ici de la terminologie de A. Weil qui n'appelle sous-variétés que les objets qui correspondent à celles des sous-varietés en notre sens qui sont fermées (cf. l'introduction). On notera que toute partie ouverte non vide de $E$ est l'ensemble des points d'une sous-variété de $E$.

Si $A$ est une sous-variété de $E$, la structure de variété de $A$ est uniquement déterminée par la connaissance de l'ensemble $A$; par abus de langage, on dit qu'une partie de $E$ est une sous-variété si c'est l'ensemble des points d'une sous-variété.

Toute sous-variété ouverte de $E$ est la réunion d'un nombre fini de sousvariétés affines ouvertes de $E$. Si $V$ est une sous-variété affine ouverte de $E$, il y a un anneau affine $\mathfrak{B}$ bien determiné du corps $\Re(E)$ tel que les anneaux locaux des points de $V$ soient les anneaux locaux de tous les idéaux maximaux de $\mathfrak{B}$; on dit que $\mathfrak{B}$ est l'anneau affine de $V$. L'anneau $\mathfrak{B}$ est l'ensemble des fonctions sur $E$ qui sont définies en tous les points de $V$.

Soit $E$ une variété affine d'anneau $\mathfrak{B}$. Pour qu'une partie $A$ de $E$ soit 
fermée, il faut et suffit qu'il existe des fonctions $f_{1}, \ldots, f_{m}$ de $\mathfrak{B}$ telles que $A$ soit l'ensemble des points $P \in E$ en lesquels $f_{1}, \ldots, f_{m}$ prennent la valeur 0 . En effet, si $A$ est fermé, son complémentaire $U$ est ouvert; soit $\mathfrak{f}(A)$ l'intersection des idéaux maximaux de $\mathfrak{B}$ dont les anneaux locaux sont des anneaux locaux de points de $A$; $A$ est alors l'ensemble des points de $E$ dont les anneaux locaux sont des anneaux locaux d'idéaux maximaux de $\mathfrak{B}$ contenant $\mathfrak{f}(A)$ (cf. la démonstration du théorème 1 , partie 6$)$. Si $\left\{f_{1}, \ldots, f_{m}\right\}$ est un ensemble de générateurs de $\mathrm{f}(A), A$ est l'ensemble des $P \in E$ tels que $f_{i}(P)=0(1 \leqq i \leqq m)$. L'idéal $\mathrm{f}(A)$ s'appelle lidéal de définition de $A$. Réciproquement, supposons la condition satisfaite. Les fonctions $f_{i}$ étant partout définies sur $E, A$ est l'intersection des complémentaires des ensembles ouverts $D\left(f_{i}^{-1}\right)(1 \leqq i \leqq m)$, donc est fermé. Pour que la partie fermée $A$ de $E$ soit une sous-variété, il est manifestement nécessaire et suffisant que $A$ soit irréductible. Pour qu'il en soit ainsi, il faut et suffit que $\mathrm{f}(A)$ soit premier. En effet, si $A$ est irréductible, $\mathfrak{f}(A)$ est l'intersection de $\mathfrak{B}$ avec l'ensemble $\mathfrak{p}$ des fonctions de $\mathfrak{R}(E)$ de trace nulle sur $A$, et nous savons que $\mathfrak{p}$ est un idéal premier de l'anneau $\mathfrak{D}(A)$ défini plus haut. Comme $\mathfrak{B} \subset \mathfrak{b}(A), \mathfrak{f}(A)=\mathfrak{p} \cap \mathfrak{B}$ est premier. Par ailleurs, si $A$ n'est pas irréductible, soit $A=A_{1} \cup A_{2}$, où $A_{1}, A_{2}$ sont des ensembles fermés $\neq A$. On a donc $\mathfrak{f}\left(A_{i}\right) \neq \mathfrak{f}(A)(i=1,2)$; par ailleurs, si $\mathfrak{M}$ est un idéal maximal qui contient $\mathrm{f}(A)$, son anneau local est l'anneau local d'un point de $A$, et ce point appartient soit à $A_{1}$ soit à $A_{2}$, de sorte que l'un au moins des idéaux $\mathrm{f}\left(A_{1}\right)$, $\mathfrak{f}\left(A_{2}\right)$ est contenu dans $\mathfrak{M}$, d'où $\mathfrak{f}\left(A_{1}\right) \mathfrak{f}\left(A_{2}\right) \subset \mathfrak{M}$. Puisque $\mathfrak{f}(A)$ est l'intersection des idéaux maximaux qui le contiennent, on a $\mathfrak{f}\left(A_{1}\right) \mathfrak{f}\left(A_{2}\right) \subset \mathfrak{f}(A)$, et $\mathfrak{f}(A)$ n'est pas premier. Observons enfin que tout idéal premier $\mathfrak{p}$ de $\mathfrak{B}$ peut se mettre sous la forme $\mathrm{f}(A)$, pour une certaine sous-variété $A$ de $V$. Il suffit pour l'établir de montrer que $\mathfrak{p}$ est l'intersection des idéaux maximaux qui le contiennent. Or, $\mathfrak{B} / \mathfrak{p}$ est un domaine d'intégrité $\mathfrak{B}$ qui est engendré par adjonction d'un nombre fini d'éléments à $\Omega$; si $x$ est un élément de $\mathfrak{B}$ non contenu dans $\mathfrak{p}$, la classe $x^{*}$ de $x$ modulo $\mathfrak{p}$ est $\neq 0$, et on sait qu'il y a alors un idéal maximal $\mathfrak{M}^{*}$ de $\mathfrak{W}$ ne contenant pas $x^{*}$; on peut écrire $\mathfrak{M}^{*}=\mathfrak{N} / \mathfrak{p}$, où $\mathfrak{M}$ est un idéal maximal de $\mathfrak{B}$ contenant $\mathfrak{p}$ mais ne contenant pas $x$, ce qui démontre notre assertion.

Proposition 1. Pour qu'une partie A d'une variété E soit une sous-variété, il faut et suffit que les conditions suivantes soient satisfaites: a) A est irréduc- 
tible; b) pour tout $P \in A$, il y a un voisinage $U$ de $P$ dans $E$ et un certain nombre de fonctions $f_{1}, \ldots, f_{m}$ définies en tout point de $U$ telles que $A \cap U$ soit l'ensemble des points $P^{\prime} \in U$ tels que $f_{i}\left(P^{\prime}\right)=0(1 \leqq i \leqq m)$.

Supposons que $A$ soit une sous-variété. Alors $A$ est l'intersection de son adhérence $\bar{A}$ avec un ensemble ouvert $V$ de $E$. Si $P \in A$, il y a une sousvariété affine ouverte $U$ de $E$ contenant $P$ et contenue dans $V$. L'ensemble $A \cap U$ étant relativement fermé dans $U$, on voit que la condition $b$ ) est satisfaite. Supposons maintenant les conditions $a$ ), $b$ ) satisfaites. Les notations étant celles de la condition $b$ ), $A \cup U$ est lintersection de $U$ et des complémentaires des ensembles ouverts $D\left(f_{i}^{-1}\right)(1 \leqq i \leqq m)$ (on peut évidemment supposer les $\left.f_{i} \neq 0\right)$; $A \cap U$ est donc relativement fermé dans $U$. Si $U_{1}$ est l'intérieur de $U, A \cap U_{1}$ est relativement fermé dans $U_{1}$, d'où $A \cap U_{1}=\bar{A} \cap U_{1}$ puisque $U_{1}$ est ouvert. Il résulte tout de suite de là que $A$ est relativement ouvert dans $\bar{A}$; étant irréductible, c'est une sous-variété.

Soient $E$ et $F$ des variétés. Nous allons définir une structure de variété sur le produit cartésien $E \times F$. Le corps $\Omega$ étant algébriquement clos, il est bien connu que l'algèbre $\Re(E) \otimes \Re(F)$ est un domaine d'intégrité; soit $\Re$ son corps des quotients. Si $\mathfrak{A}$ et $\mathfrak{B}$ sont des sous-espaces vectoriels de $\mathfrak{R}(E)$ et $\mathfrak{R}(F)$ sur $\Omega$, leur produit tensoriel $\mathfrak{R} \otimes \mathfrak{B}$ s'identifie à un sous-espace de $\mathfrak{R}$. Soient $P$ un point de $E$ et $Q$ un point de $F$; il y a alors un homomorphisme $\varphi_{P, Q}$ de $\mathfrak{o}(P) \otimes \mathfrak{o}(Q)$ sur $\Omega$ qui applique $f \otimes g$ sur $f(P) g(Q)$ si $f \in \mathfrak{o}(P), g \in \mathfrak{o}(Q)$; le noyau $\mathfrak{m}_{P, Q}$ de cet homomorphisme est $\mathfrak{m}_{P} \otimes \mathfrak{D}(Q)+\mathfrak{D}(P) \otimes \mathfrak{m}_{Q}$, où $\mathfrak{m}_{P}$, $\mathfrak{m}_{Q}$ sont les idéaux maximaux de $\mathfrak{D}(P)$ et $\mathfrak{v}(Q)$. Nous désignerons par $\mathfrak{o}(P, Q)$ l'anneau local de l'idéal premier $m_{P, Q}$. Il est clair que $\varphi_{P, Q}$ se prolonge en un homomorphisme, que nous désignerons encore par $\varphi_{P, Q}$, de $\mathfrak{D}(P, Q)$ sur $\Omega$ tel que $\varphi_{P, Q}\left(u v^{-1}\right)=\varphi_{P, Q}(u)\left(\varphi_{P, Q}(v)\right)^{-1}$, si $u$, $v$ sont dans $\mathfrak{D}(P) \otimes \mathfrak{D}(Q)$ et si $v$ n'est pas dans $\mathrm{m}_{P, Q}$. Si $u$ est un élément quelconque de $\Re$, nous désignerons par $D(u)$ l'ensemble des points $(P, Q)$ tels que $u \in \mathfrak{o}(P, Q)$ et par $\theta(u)$ l'application $(P, Q) \rightarrow \varphi_{P, Q}(u)$ de $D(u)$ dans $\Omega$. Si $u, v$ sont des éléments de $\Re$, et si $(P, Q)$ $\in D(u) \cap D(v)$, on a $(P, Q) \in D(u+v), \quad(\theta(u+v))(P, Q)=(\theta(u))(P, Q)$ $+(\theta(v))(P, Q)$. Soit réciproquement $w$ un élément de $\Re$ tel que $D(w)$ $\supset D(u) \cap D(v)$ et que $(\theta(w))(P, Q)=(\theta(u))(P, Q)+(\theta(v))(P, Q)$ pour tout point $(P, Q)$ de $D(u) \cap D(v)$. Soient $U, V$ des sous-variétés affines ouvertes de $E, F$, et $\mathfrak{U}, \mathfrak{Z}$ leurs anneaux. Il est alors clair que $\mathfrak{R}$ est le corps des quotients de 
$\mathfrak{u} \otimes \mathfrak{B}$. Montrons que, pour tout idéal maximal $\mathfrak{M}$ de $\mathfrak{u} \otimes \mathfrak{B}$, l'anneau local $\mathfrak{o}$ de $\mathfrak{M}$ peut se mettre sous la forme $\mathfrak{o}(P, Q)$, avec $P \in E, Q \in F$. L'ensemble $\mathfrak{M}_{E}$ des $f \in \mathfrak{u}$ tels que $f \otimes 1 \in \mathfrak{M}$ est un idéal. L'homomorphisme $f \rightarrow f \otimes 1$ de $\mathfrak{U}$ dans $\mathfrak{u} \otimes \mathfrak{B}$ définit un isomorphisme de $\mathfrak{H} / \mathfrak{M}_{F}$ sur un sous-anneau de $(\mathfrak{H} \otimes \mathfrak{B}) / \mathfrak{M}$. Or $\mathfrak{U} \otimes \mathfrak{V}$ est manifestement un anneau affine sur $\Omega ;$ il en résulte que $(\mathfrak{H} \otimes \mathfrak{B}) / \mathfrak{M}=\Omega$, donc que $\mathfrak{H} / \mathfrak{M}_{E}=\Omega$. L'idéal $\mathfrak{M}_{E}$ est donc maximal. On voit de même que l'ensemble $\mathfrak{M}_{F}$ des $g \in \mathfrak{B}$ tels que $1 \otimes g \in \mathfrak{R}$ est un idéal maximal. Il y a donc un point $(P, Q) \in E \times F$ tel que $\mathfrak{D}(P)$ et $\mathfrak{o}(Q)$ soient les anneaux locaux de $\mathfrak{M}_{E}$ et de $\mathfrak{M}_{F}$. Le noyau $\mathfrak{M}_{E} \otimes \mathfrak{B}+\mathfrak{H} \otimes \mathfrak{D}_{F}$ de la restriction de $\varphi_{P, \ell}$ à $\mathfrak{l} \otimes \mathfrak{B}$ est contenu dans $\mathfrak{R}$ et est un idéal maximal; il est donc identique à $\mathfrak{M}$, et il en résulte immédiatement que $\mathfrak{o}(P) \otimes \mathfrak{b}(Q) \subset \mathfrak{b}$. Puisque $\mathfrak{H} \otimes \mathfrak{B}$ $\mathcal{C}(P) \otimes \mathfrak{D}(Q) \subset_{\mathfrak{D}}$ et puisque $\mathfrak{D}$ est l'anneau local d'un idéal premier de $\mathfrak{H} \otimes \mathfrak{B}$, on voit tout de suite que c'est l'anneau local d'un idéal premier de $\mathfrak{D}(P) \otimes \mathfrak{D}(Q)$, intersection de cet anneau avec l'idéal maximal de $\mathfrak{D}$. Soit $\mathrm{m}^{\prime}$ cet idéal; $\mathrm{m}_{P}$ et $\mathfrak{m}_{Q}$ étant engendrés par $\mathfrak{M}_{E}$ et $\mathfrak{M}_{F}$ respectivement, il est clair que $\mathfrak{m}^{\prime}$ contient $\mathrm{m}_{P, Q}$, donc lui est identique, puisque $\mathrm{m}_{P, Q}$ est maximal. On a donc bien $\mathfrak{o}=\mathfrak{o}(P, Q)$. Ceci dit, on peut écrire $u=u_{1} u_{2}^{-1}, v=v_{1} v_{2}^{-1}, w=w_{1} w_{2}^{-1}$, où $u_{1}, u_{2}, v_{1}, v_{2}, w_{1}, w_{2}$ sont dans $\mathfrak{U} \otimes \mathfrak{B}$ et $u_{2} v_{2} w_{2} \neq 0 . \quad$ Si $u_{2} v_{2} w_{2}$ n'est pas dans $\mathfrak{M}$, on a $(P, Q)$ $\in D(u) \cap D(v)$, et il résulte de l'hypothèse faite sur $w$ que $(\theta(w))(P, Q)$ $=(\theta(u))(P, Q)+(\theta(v))(P, Q)$, donc que l'élément $u_{2} v_{2} w_{2}(w-(u+v))$ de $\mathfrak{u} \otimes \mathfrak{B}$ est dans $\mathfrak{M}$. Or, $\mathfrak{u} \otimes \mathfrak{B}$ étant un anneau affine, si $u_{2} v_{2} w_{2}(w-(u+v))$ n'était pas nul, il y aurait un idéal maximal $\mathfrak{M}$ de $\mathfrak{U} \otimes \mathfrak{B}$ qui ne contiendrait pas cet élément: c'est impossible, comme on vient de le voir. On a donc $w=u+v$. On voit de même que le seul élément $t \in \mathfrak{R}$ tel que $D(u) \cap D(v) \subset D(t)$ et que $(\theta(t))(P, Q)=(\theta(u))(P . Q)(\theta(v))(P, Q)$ pour tout $(P, Q) \in D(u) \cap D(v)$ est $u v$. Nous désignerons par $\Re(E \times F)$ l'ensemble des fonctions $\theta(u), u \in \Re$. Il résulte de ce que nous venons de dire que $\theta$ est une application biunivoque de $\Re$ sur $\Re(E \times F)$; cette application transporte à $\Re(E \times F)$ la structure de corps de $\Re$. Les conditions I, II, III du début de II sont manifestement satisfaites pour $E \times F$ et $\Re(E \times F)$.

Reprenons les notations utilisées plus haut. Nous allons montrer que réciproquement, si $(P, Q)$ est un point quelconque de $U \times V, \mathfrak{o}(P, Q)$ est l'anneau local d'un idéal maximal de $\mathfrak{U} \otimes \mathfrak{B}$. On a $\mathfrak{u} \otimes \mathfrak{B} \subset \mathfrak{D}(P) \otimes \mathfrak{D}(Q) \subset \mathfrak{D}(P, Q)$; l'homomorphisme $\varphi_{P, Q}$ de $\mathfrak{b}(P, Q)$ sur $\Omega$ induit un homomorphisme de $\mathfrak{u} \otimes \mathfrak{V}$ sur $\Omega$ dont le noyau est un idéal maximal $\mathfrak{M}$. L'anneau local de $\mathfrak{M}$ est de la 
forme $\mathfrak{D}\left(P^{\prime}, Q^{\prime}\right)$, où $P^{\prime}$ est un point de $U$ et $Q^{\prime}$ un point de $V$; de plus, si $\mathfrak{I}_{E}$, $\mathfrak{M}_{F}$ sont les idéaux maximaux de $\mathfrak{l}, \mathfrak{B}$ dont les anneaux locaux sont $\mathfrak{D}\left(P^{\prime}\right)$, $\mathfrak{o}\left(Q^{\prime}\right), \mathfrak{M}_{E}$ est l'ensemble des $f \in U$ tels que $f \otimes I \in \mathfrak{M}$, donc tels que $f(P)=0$, ce qui montre que $P=P^{\prime}$, et on voit de même que $Q=Q^{\prime} ; \mathfrak{o}(P, Q)$ est donc l'anneau local de $\mathfrak{M}$. L'ensemble des $o(P, Q)$ pour tous les $(P, Q) \in U \times V$ est donc l'ensemble des anneaux locaux des idéaux maximaux de $\mathfrak{H} \otimes \mathfrak{B}$. Or, la variété $E$ est la réunion d'un nombre fini de sous-variétés ouvertes affines $U_{i}(1 \leqq i \leqq h)$ et $F$ d'un nombre fini de sous-variétés ouvertes affines $V_{j}$ $(1 \leqq j \leqq k) ; E \times F$ est donc la réunion des ensembles $U_{i} \times V_{j}(1 \leqq i \leqq h ; 1 \leqq j \leqq k)$. Il ne reste donc qu'à montrer que, si $(P, Q)$ et $\left(P^{\prime}, Q^{\prime}\right)$ sont des points distincts de $E \times F$, les localités $o(P, Q)$ et $o\left(P^{\prime}, Q^{\prime}\right)$ ne se correspondent pas. Supposons par exemple que $P \neq P^{\prime}$, et soit 0 l'anneau engendré par $\mathfrak{o}(P)$ et $\mathfrak{o}\left(P^{\prime}\right)$. Les éléments $f \otimes 1, f \in \mathfrak{D}$, forment alors un sous-anneau de l'anneau engendré par $\mathfrak{D}(P, Q)$ et $\mathfrak{D}\left(P^{\prime}, Q^{\prime}\right)$. Puisque $P \neq P^{\prime}$, l'idéal engendré par $m_{P}$ et $m_{P^{\prime}}$ dans $\mathfrak{D}$ contient 1 ; puisque $m_{P} \otimes\{1\} \subset \mathrm{m}_{P, Q}, \mathrm{~m}_{P^{\prime}} \otimes\{1\} \subset \mathrm{m}_{P^{\prime}, \psi^{\prime}}$ on en conclut que l'idéal engendré par $\mathfrak{m}_{P, Q}$ et $\mathfrak{m}_{P^{\prime}, Q^{\prime}}$ dans l'anneau engendré par $\mathfrak{D}(P, Q)$ et $\mathfrak{D}\left(P^{\prime}, Q^{\prime}\right)$ contient 1.

Le corps $\Re(E \times F)$ des fonctions sur $E \times F$ est, comme nous lavons vu, canoniquement isomorphe au corps des quotients $\mathfrak{R}$ de $\Re(E) \otimes \mathfrak{H}(F)$; on identifiera en général $\Re(E \times F)$ à $\Re$.

La variété $E \times F$ s'appelle le produit des variétés $E, F$. On a vu au cours de la démonstration que le produit d'une sous-variété affine ouverte de $E$, d'anneau $\mathfrak{H}$, par une sous-variété affine ouverte de $F$, d'anneau $\mathfrak{B}$, est une sousvariété affine ouverte de $E \times F$, d'anneau $\mathfrak{U} \otimes \mathfrak{B}$. Toute partie ouverte d'une variété étant réunion de sous-variétés affines ouvertes, il en résulte immédiatement que les projections de $E \times F$ sur $E$ et sur $F$ sont continues (au sens de la topologie de Zariski). Mais la topologie de Zariski de $E \times F$ est en généra! différente du produit des topologies de Zariski de $E$ et de $F$, comme on le voit déjà dans le cas où $E, F$ sont des espaces affines de dimension 1.

PRoposition 2. Soit u une fonction sur le produit de deux variétés $E$ et $F$, et soit $(P, Q)$ un point de $E \times F$ en lequel u est définie. L'ensemble $V$ des points $Q^{\prime} \in F$ en lesquels $u$ est définie est alors ouvert, et l'application $Q^{\prime} \rightarrow u\left(P, Q^{\prime}\right)$ de cet ensemble dans $\Omega$ est la restriction à $V$ d'une fonction sur $F$.

On peut représenter $u$ comme quotient de deux fonctions $u_{1}, u_{2}$ appartenant 
à $\mathfrak{D}(P) \otimes \mathfrak{o}(Q)$ de telle manière que $u_{2}(P, Q) \neq 0$. Posons $u_{1}=\sum_{i=1}^{m} f_{i} \otimes g_{i}, u_{2}$ $=\sum_{j=1}^{n} f_{j}^{\prime} \otimes g_{j}^{\prime}$, les $f_{i}, f_{j}^{\prime}$ étant dans $\mathfrak{o}(P)$ et les $g_{i}, g_{j}^{\prime}$ dans $\mathfrak{o}(Q)$. La fonction $\sum_{j=1}^{n} f_{j}^{\prime}(P) g_{j}^{\prime}=g^{\prime}$ est $\neq 0$ et $g^{\prime-1}$ est définie en $Q$. L'ensemble $V^{\prime}$ des points de $F$ en lesquels $g_{1}, \ldots, g_{m}, g_{1}^{\prime}, \ldots, g_{n}^{\prime}, g^{1^{-1}}$ sont toutes définies est ouvert et contient $Q$; il est clair que cet ensemble est contenu dans $V$ et que la restriction à cet ensemble de l'application $Q^{\prime} \rightarrow u\left(P, Q^{\prime}\right)$ est aussi la restriction d'une fonction $h^{\prime}$ sur $F$. Il résulte de là que tout point de $V$ est contenu dans un ensemble ouvert de $F$ contenu dans $V$, donc que $V$ est ouvert. De plus, si $h^{\prime \prime}$ est une fonction quelconque de $\Re(F)$ et $V^{\prime \prime}$ une partie ouverte non vide de $V$ telle que $h^{\prime \prime}$ soit définie sur $V^{\prime \prime}$ et que $h^{\prime \prime}\left(Q^{\prime}\right)=u\left(P, Q^{\prime}\right)$ pour tout $Q^{\prime} \in V^{\prime \prime}, h^{\prime \prime}$ coïncide avec $h^{\prime}$ sur l'ensemble ouvert $V^{\prime} \cap V^{\prime \prime} ; F$ étant irréductible, $V^{\prime} \cap V^{\prime \prime}$ est dense dans $F$, et il en résulte que $h^{\prime}=h^{\prime \prime}$. La prop. 2 est donc démontrée.

On aurait évidemment une proposition analogue à celle que nous venons de démontrer en échangeant les rôles joués par $E$ et $F$.

Soient $E$ et $F$ des variétés, et $\pi_{F}, \pi_{F}$ les projections de $E \times F$ sur $E$ et sur $F$. Si $P \in E$, l'ensemble $\{P\} \times F$, image inverse de $\{P\}$ par rapport à $\pi_{E}$, est fermé. La restriction de $\pi_{F}$ à cet ensemble est une application continue biunivoque de $\{P\} \times F$ sur $F$. Montrons que c'est un homéomorphisme. Il suffit de montrer que l'image d'une partie relativement ouverte de $\{P\} \times F$ est ouverte dans $F$. Or la topologie de $E \times F$ est engendrée par les ensembles $D(u)$ $(u \in \Re(E \times F)$ ), où $D(u)$ est l'ensemble des points en lesquels $u$ est définie; et il résulte de la proposition 2 que l'image par $\pi_{F}$ de $D(u) \cap(\{P\} \times F)$ est ouverte dans $F$; ceci démontre notre assertion.

Proposition 3. Soient $E$ et $F$ des variétés, $A$ une sous-variété de $E$ et $B$ une sous-variété de $F$. La variété produit $A \times B$ est alors une sous-variété de $E \times F$.

Montrons d'abord que $A \times B$ est irréductible dans $E \times F$. Soit $A \times B=C_{1} \cup C_{2}$ une représentation de cet ensemble comme réunion de deux parties relativement fermées $C_{1}, C_{2}$. Soit $P$ un point de $A$; désignons par $B_{i}(P)$ l'ensemble des $Q \in B$ tels que $(P, Q) \in C_{i}(i=1,2) ;$ on a $B=B_{1}(P) \cup B_{2}(P)$. Lensemble $B_{i}(P)$ est la projection sur $F$ de l'ensemble $C_{i} \cap(\{P\} \times F)$, qui est relativement fermé dans $\{P\} \times B$. Puisque la projection sur $F$ induit un homéomorphisme 
de $\{P\} \times F, B_{i}(P)$ est relativement fermé dans $B$. L'ensemble $B$ étant irréductible, l'un des ensembles $B_{1}(P), B_{2}(P)$ est $B$ tout entier. Soit $A_{i}$ l'ensemble des $P \in A$ tels que $\{P\} \times B \subset C_{i}(i=1,2)$; on a donc $A_{1} \cup A_{2}=A$. Or, $A_{i}$ est l'intersection pour tous les $Q \in B$ des ensembles $A_{i}(Q)$, où $A_{i}(Q)$ est lensemble des $P \in A$ tels que $(P, Q) \in C_{i}$; on voit comme plus haut que les $A_{i}(Q)$ sont tous relativement fermés dans $A$, donc qu'il en est de même de $A_{i}$. Comme $A$ est irréductible, l'un des ensembles $A_{1}, A_{2}$ est $A$ tout entier; si $A_{i}=A$, on a $C_{i}=A \times B ; A \times B$ est donc bien irréductible.

Soit $u$ une fonction sur $E \times F$ qui a une trace $u^{*}$ sur $A \times B$; nous nous proposons de montrer que $u^{*}$ est une fonction sur la variété produit $A \times B$. Supposons $u$ définie en un point $(P, Q)$ de $A \times B$. Écrivons $u=u_{1} u_{2}^{-1}, u_{1}$ $=\sum_{i=1}^{m} f_{i} \otimes g_{i}, u_{2}=\sum_{j=1}^{n} f_{j}^{\prime} \otimes g_{j}^{\prime}$, où les $f_{i}, f_{j}^{\prime}$ sont dans $\mathrm{D}(P)$, les $g_{i}, g_{j}^{\prime}$ dans $\mathrm{o}(Q)$ et où $\sum_{j=1}^{n} f_{j}^{\prime}(P) g_{j}^{\prime}(Q) \neq 0$. Les fonctions $f_{i}, f_{j}^{\prime}$ ont donc des traces $F_{i}, F_{j}^{\prime}$ sur $A$, les fonctions $g_{i}, g_{j}^{\prime}$ des traces $G_{i}, G_{j}^{\prime}$ sur $B$; on a $\sum_{j=1}^{n} F_{j}^{\prime} \otimes G_{j}^{\prime} \neq 0$ et le quotient $H$ de $\sum_{i=1}^{m} F_{i} \otimes G_{i}$ par $\sum_{j=1}^{n} F_{j}^{\prime} \otimes G_{j}^{\prime}$ est défini en $(P, Q)$. Soit $W_{1}$ l'ensemble des points de $A \times B$ en lesquels $\left(\sum_{j=1}^{n} F_{j}^{\prime} \otimes G_{j}^{\prime}\right)^{-1}$ est définie. Soit $U$ l'ensemble des points de $A$ en lesquels $f_{1}, \ldots, f_{m}, f_{1}^{\prime}, \ldots, f_{n}^{\prime}$ sont tous définis, et soit $V$ l'ensemble des points de $B$ en lesquels $g_{1}, \ldots, g_{m}, g_{1}^{\prime}, \ldots, g_{n}^{\prime}$ sont tous définis. Soit $\left(P^{\prime}, Q^{\prime}\right)$ un point de $W_{1} \cap(U \times V)$; les fonctions $F_{i}$, $F_{j}^{\prime}$ sont alors définies en $P^{\prime}$, les $G_{i}, G_{j}^{\prime}$ sont définis en $Q^{\prime}$, on a $f_{i}\left(P^{\prime}\right)=F_{i}\left(P^{\prime}\right), f_{j}^{\prime}\left(P^{\prime}\right)$ $=F_{j}^{\prime}\left(P^{\prime}\right), \quad g_{i}\left(Q^{\prime}\right)=G_{i}\left(Q^{\prime}\right), g_{j}^{\prime}\left(Q^{\prime}\right)=G_{j}^{\prime}\left(Q^{\prime}\right) ;$ puisque $\left(P^{\prime}, Q^{\prime}\right) \in W_{1}$, on a $\sum_{j=1}^{n} f_{j}^{\prime}\left(P^{\prime}\right) g_{j}^{\prime}\left(Q^{\prime}\right) \neq 0$. La fonction $u$ est donc définie en $\left(P^{\prime}, Q^{\prime}\right)$; il en est de même de $u^{*}$ et de $H$, et on a $u\left(P^{\prime}, Q^{\prime}\right)=u^{*}\left(P^{\prime}, Q^{\prime}\right)=H\left(P^{\prime}, Q^{\prime}\right)$. Les topologies de Zariski de $A$ et de $B$ étant induites par celles de $E, F$ respectivement, $U$ et $V$ sont ouverts dans ces topologies. Les projections de $A \times B$ sur $A$ et sur $B$ étant continues relativement à la topologie de Zariski $\mathfrak{I}$ de la variété produit $A \times B, U \times V$ est ouvert dans $\mathfrak{I}$, et il en est de même de $(U \times V) \cap W_{1}$. De plus, ce dernier ensemble contient $(P, Q)$. Si $(P, Q)$ est un point quelconque en lequel $u^{*}$ est défini, $u^{*}$ est la trace sur $A \times B$ d'une fonction $u$ sur $E \times F$ définie en $(P, Q)$; on en conclut qu'il y a alors une partie ouverte $W$ (pour la topologie $\mathfrak{I})$ de $A \times B$ sur laquelle $u^{*}$ est définie et coüncide avec une fonction sur la variété produit. L'intersection de deux parties ouvertes non vides de $A \times B$ (relativement à $\mathfrak{I}$ ) est dense dans $A \times B$; deux fonctions sur $A \times B$ 
qui sont définies et coïncident sur cette intersection sont donc égales. Il en résulte immédiatement qu'il y a une fonction $H$ sur la variété produit qui est définie en tous les points où $u^{*}$ est définie et qui prolonge $u^{*}$; de plus, l'ensemble des points de $A \times B$ où $u^{*}$ est défini est ouvert dans la topologie $\mathfrak{T}$.

Partons maintenant réciproquement d'une fonction $H$ quelconque sur la variété produit $A \times B$, et soit $(P, Q)$ un point en lequel cette fonction est définie. Nous pouvons mettre la fonction $H$ sous la forme $\left(\sum_{i=1}^{m} F_{i} \otimes G_{i}\right)\left(\sum_{j=1}^{n} F_{j}^{\prime} \otimes G_{j}^{\prime}\right)^{-1}$, où les $F_{i}, F_{j}^{\prime}$ sont des fonctions sur $A$ définies en $P$ et les $G_{i}, G_{j}^{\prime}$ des fonctions sur $B$ définies en $Q$ et $\sum_{j=1}^{n} F_{j}^{\prime}(P) G_{j}^{\prime}(Q) \neq 0$. On peut représenter les $F_{i}, F_{j}^{\prime}$ comme des traces sur $A$ de fonctions $f_{i}, f_{j}^{\prime}$ sur $E$ définies en $P$ et les $G_{i}$, $G_{j}^{\prime}$ comme des traces sur $B$ de fonctions $g_{i}, g_{j}^{\prime}$ définies en $Q$. Posons $u=\left(\sum_{i=1}^{m} f_{i} \otimes g_{i}\right)\left(\sum_{j=1}^{n} f_{j}^{\prime} \otimes g_{j}^{\prime}\right)^{-1} ; u$ est une fonction sur $E \times F$ définie en $(P, Q)$. Soit $u^{*}$ sa trace sur $A \times B$. Définissons les ensembles $U, V$ comme plus haut, et soit $W_{1}^{\prime}$ l'ensemble des points de $A \times B$ en lesquels $\left(\sum_{j=1}^{n} f_{j}^{\prime} \otimes g_{j}^{\prime}\right)^{-1}$ est défini. On voit alors tout de suite que $u^{*}$ et $H$ sont partout définis et coïncident sur l'ensemble $(U \times V) \cap W_{1}^{\prime}$, qui contient $(P, Q)$, et coïncident l'une avec l'autre sur cet ensemble. Soit $\mathfrak{I}^{\prime}$ la topologie induite sur $A \times B$ par la topologie de Zariski de $E \times F$. Les ensembles $U$ et $V$ étant relativement ouverts dans $A$ et $B$ respectivement et les projections de $E \times F$ sur $E$ et $F$ étant continues, il est clair que $U \times V$ est ouvert dans $\mathfrak{I}^{\prime}$. Il en est évidemment de même de $W_{1}^{\prime}$; l'ensemble $(U \times V) \cap W_{1}^{\prime}$ est donc ouvert dans $\mathfrak{I}^{\prime}$. Puisque $A \times B$ est irréductible dans $E \times F$, si les traces sur $A \times B$ de deux fonctions sur $E \times F$ sont définies et coïncident sur un ensemble non vide ouvert dans la topologie $\mathfrak{I}^{\prime}$, ces traces coïncident. On voit alors de la même manière que plus haut qu'il y a une fonction sur $E \times F$ dont la trace sur $A \times B$ est définie en tous les points du domaine de définition de $H$ et prolonge $H$. Or, si deux fonctions sur une même variété sont telles que l'une soit une restriction de l'autre, ces fonctions sont manifestement identiques (leur différence étant nulle sur un ouvert non vide). On en conclut que les fonctions sur la variété produit $A \times B$ sont identiques aux traces sur $A \times B$ de celles des fonctions sur $E \times F$ qui ont des traces sur cet ensemble, ce qui démontre la proposition 3 .

On appelle correspondance irréductible entre les variétés $E$ et $F$ toute sousvariété $T$ du produit $E \times F$. Etant donnée une correspondance irréductible $T$ 
entre $E$ et $F$, on dit qu'un point $Q$ de $F$ correspond à un point $P$ de $E$ par $T$ si $(P, Q) \in T$. La projection de $T$ sur $E$ s'appelle le domaine de la correspondance $T$, et sa projection sur $F$ le contre-domanie de $T$.

Proposition 4. Le domaine et le contre-domaine d'une correspondance irréductible entre deux variétés sont des ensembles irréductibles.

Cela résulte immédiatement du

Lemme. Soit $H$ une application continue d'un espace de Zariski $A$ dans un espace de Zariski $B$. Si $C$ est une partie irréductible de $A, H(C)$ est une partie irréductible de $B$.

Soient $D_{1}, D_{2}$ des parties relativement fermées de $H(C)$ dont la réunion est $H(C)$; soit $C_{i}$ l'ensemble des $P \in C$ tels que $H(P)=D_{i}$. 'Les ensembles $C_{1}, C_{2}$ sont relativement fermés dans $C$ puisque $H$ est continue, et leur réunion est $C$. L'un de ces ensembles est donc $C$ tout entier, et l'un des ensembles $D_{1}, D_{2}$ est $H(C)$.

Nous examinerons plus tard de manière plus précise la nature du domaine et du contre-domaine d'une correspondance irréductible.

Une correspondance irréductible $T$ entre des variétés $E$ et $F$ est dite non dégénérée si les conditions suivantes sont satisfaites: le domaine de $T$ est dense dans $E$ et son contre-domaine est dense dans $F$. S'il n'en est pas ainsi, soient $A$ et $B$ les adhérences du domaine et du contre-domaine de $T$; ce sont des sous-variétés de $E$ et de $F$ (en vertu de la proposition 4), et $T$ est une correspondance irréductible entre $A$ et $B$ (en vertu de la proposition 3). L'étude des correspondances irréductibles quelconques peut donc se ramener à l'étude de celles qui sont non dégénérées.

Soient $\Re / \Omega, \Re^{\prime} / \Omega$ deux extensions de $\Omega$. On appelle extension composée de ces deux extensions un système $\left(\Omega, \lambda, \lambda^{\prime}\right)$ composé d'une extension $\Omega / \Omega$ de $\Omega$ et d'isomorphismes $\lambda$ de $\Re$ et $\lambda^{\prime}$ de $\Re^{\prime}$ sur des sous-corps de $\Omega$ qui possèdent les propriétés suivantes: $\lambda$ et $\lambda^{\prime}$ coïncident avec l'identité sur $\Omega ; \mathcal{L}$ est engendré par les corps $\lambda(\Re)$ et $\lambda^{\prime}\left(\Re^{\prime}\right)$. Deux extensions composées $\left(\mathscr{L}, \lambda, \lambda^{\prime}\right)$ et $\left(\Omega_{1}, \lambda_{1}\right.$, $\left.\lambda_{1}^{\prime}\right)$ de $\Re / \Omega$ et $\Re^{\prime} / \Omega$ sont dites isomorphes s'il existe un isomorphisme $\theta$ de $\mathscr{Z}$ sur $\Omega_{1}$ tel que $\lambda_{1}=\theta \circ \lambda$ et $\lambda_{1}^{\prime}=\theta \circ \lambda^{\prime}$.

Généralisant la définition que nous avons donnée pour les localités d'un corps, nous dirons que deux sous-anneaux $\mathfrak{o}$ et $\mathfrak{b}^{\prime} \mathrm{d}^{\prime} u n$ même corps qui sont des 
anneaux locaux (i.e. dans chacun de ces anneaux, les non-unités forment un idéal, qui est alors l'unique idéal maximal de l'anneau) se correspondent si lidéal engendré par les idéaux maximaux de $\mathfrak{D}$ et $\mathfrak{b}^{\prime}$ dans l'anneau engendré par $\mathfrak{D}$ et $\mathfrak{D}^{\prime}$ ne contient pas 1 .

THÉORÈme 2. Soient $E$ et $F$ des variétés, et soit $\left(\Omega, \lambda, \lambda^{\prime}\right)$ une extension composée dos extensions $\Re(E) / \Omega, \mathfrak{R}(F) / \Omega$. A cette extension composée se trouve alors associée une correspondance irréductible non dégénérée fermée $T$ (i.e. $T$ est une sous-variété fermée de $E \times F)$ entre $E$ et $F$ qui se compose des couples $(P, Q) \in E \times F$ tels que $\lambda(\mathcal{o}(P))$ et $\lambda^{\prime}(\mathfrak{o}(Q))$ se correspondent dans $\mathbb{R} ; \Re(T)$ est isomorphe à $\mathrm{S}$. Toute correspondance irréductible non dégénérée fermée entre $E$ et $F$ peut se définir de la manière précédente. Pour que deux extensions composées de $\Re(E) / \Omega, \mathfrak{i}(F) / \Omega$ définissent la même correspondance, il faut et suffit quelles soient isomorphes.

Si $\left(R, \lambda, \lambda^{\prime}\right)$ est une extension composée de $\Re(E) / \Omega$ et de $\Re(F) / \Omega$, il existe un homomorphisme $A$ de $\Re(E) \otimes \Re(F)$ dans $\mathfrak{L}$ tel que $A(f \otimes g)=\lambda(f) \lambda^{\prime}(g)$ si $f \in \Re(E), g \in \mathfrak{R}(F)$. Soit $\mathfrak{p}$ le noyau de cet homomorphisme: c'est un idéal premier. Si $(P, Q) \in E \times F$, nous désignerons par $\mathfrak{m}_{P}, \mathfrak{m}_{Q}$ les idéaux maximaux de $\mathfrak{D}(P), \mathfrak{o}(Q)$ et par $\mathfrak{m}_{P, Q}$ l'idéal maximal $\mathfrak{m}_{P} \otimes \mathfrak{o}(Q)+\mathfrak{p}(P) \otimes \mathfrak{m}_{Q} \operatorname{de} \mathfrak{v}(P) \otimes \mathfrak{o}(Q)$. Soit $T$ l'ensemble des couples $(P, Q)$ tels que $\mathfrak{p} \cap(\mathfrak{b}(P) \otimes \mathfrak{o}(Q)) \subset \mathrm{m}_{P, Q}$. Nous nous proposons de montrer que $T$ est une partie fermée de $E \times F$. Il suffira évidemment de montrer que, si $U$ et $V$ sont des sous-variétés affines ouvertes de $E$ et de $F, T \cap(U \times V)$ est relativement fermé dans $U \times V$. Soient $\mathfrak{u}$ et $\mathfrak{B}$ les anneaux affines de $U$ et $V$; celui de $U \times V$ est alors $\mathfrak{u} \otimes \mathfrak{B}$. Supposons que $(P, Q) \in U \times V ;$ soit $\mathfrak{N}_{P}=\mathfrak{m}_{P} \cap \mathfrak{H}, \mathfrak{R}_{Q}=\mathfrak{m}_{Q} \cap \mathfrak{B} ; \mathfrak{d}(P, Q)$ est alors l'anneau local de l'idéal maximal $\mathfrak{M}_{P, Q}=\mathfrak{M}_{P} \otimes \mathfrak{B}+\mathfrak{U} \otimes \mathfrak{M}_{Q}$ de $\mathfrak{u} \otimes \mathfrak{B}$, et $\mathfrak{M}_{P, Q}=(\mathfrak{u} \otimes \mathfrak{B}) \cap \mathfrak{m}_{P, Q}$ (car $\mathfrak{m}_{P, Q}$ contient manifestement $\mathfrak{M}_{P, Q}$ et ce dernier est maximal). $\mathrm{Si}(P, Q)$ $\in T$, on a $\mathfrak{p} \cap(\mathfrak{H} \otimes \mathfrak{B}) \subset \mathfrak{M}_{P, Q}$. La réciproque est vraie. Supposons en effet que $\mathfrak{p} \cap(\mathfrak{H} \otimes \mathfrak{B})$ soit dans $\mathfrak{M}_{P, Q}$. Soit $u$ un élément de $\mathfrak{p} \cap(\mathfrak{o}(P) \otimes \mathfrak{o}(Q))$. Il est clair qu'on peut trouver des éléments $f \in \mathfrak{H}, g \in \mathfrak{B}$ tels que $f \notin \mathfrak{M}_{P}, g \notin \mathfrak{M}_{Q}$ et que $(f \otimes g) u \in \mathfrak{H} \otimes \mathfrak{B}\left(\mathfrak{D}(P)\right.$ et $\mathfrak{D}(Q)$ sont en effet les anneaux locaux de $\mathfrak{M}_{P}$ et de $\left.\mathfrak{M}_{Q}\right)$. On a aussi $(f \otimes g) u \in \mathfrak{p}$; cet élément est donc dans $\mathfrak{M}_{P, Q}$ et peut se mettre sous la forme $(f \otimes g) u=\sum_{i=1}^{m} x_{i} \otimes g_{i}+\sum_{j=1}^{n} f_{j} \otimes y_{j}$ où les $x_{i}$ sont dans $\mathfrak{M}_{P}$, les $g_{i}$ dans $\mathfrak{D}(Q)$, les $f_{j}$ dans $\mathfrak{o}(P)$ et les $y_{j}$ dans $\mathfrak{M}_{Q}$; il en résulte immédiate- 
ment que $u \in \mathfrak{m}_{P, Q}$; on a donc $\mathfrak{p} \cap(\mathfrak{D}(P) \otimes \mathfrak{D}(Q)) \subset \mathfrak{m}_{P, Q}$ et $(P, Q) \in T$. L'ensemble $(U \times V) \cap T$ est donc une sous-variété irréductible fermée de $U \times V$. De plus, les fonctions de $\mathfrak{R}(E \times F)$ qui ont des traces sur cette sous-variété sont celles de l'anneau local $\mathfrak{o}$ de l'idéal premier $\mathfrak{p} \cap(\mathfrak{U} \otimes \mathfrak{B})$ de $\mathfrak{U} \otimes \mathfrak{B}$. Cet anneau local est aussi celui de l'idéal $\mathfrak{p}$ de $\mathfrak{R}(E) \otimes \mathfrak{R}(F)$. Soient en effet $x$ et $y$ des éléments $\neq 0$ quelconques de $\mathfrak{l}$ et de $\mathfrak{B}$. Puisque $\lambda$ et $\lambda^{\prime}$ sont des isomorphismes de $\mathfrak{R}(E)$ et $\mathfrak{N}(F)$ respectivement, on a $\lambda(x) \neq 0, \lambda^{\prime}(y) \neq 0$ d'où $A(x \otimes y) \neq 0$ et $(x \otimes y)^{-1} \in 0 . \quad$ Si $f$ et $g$ sont des éléments quelconques de $\mathfrak{R}(E)$ et $\Re(F)$ respectivement, on peut écrire $f=x^{-1} x^{\prime}$, avec $x, x^{\prime} \in \mathfrak{U}$ et $g=y^{-1} y^{\prime}$, avec $y, y^{\prime} \in \mathfrak{B}$; il en résulte immédiatement que $f \otimes g \in 0$, d'où $\Re(E) \otimes \Re(F) \subset 0$. Soit q l'idéal maximal de $\mathfrak{o}$; il est engendré par $(\mathfrak{U} \otimes \mathfrak{B}) \cap \mathfrak{p}$, et il est clair que $\mathfrak{D}$ est l'anneau local de l'idéal $q \cap(\mathfrak{R}(E) \otimes \Re(F))$ de $\mathfrak{N}(E) \otimes \Re(F)$. Par ailleurs, $\mathfrak{N}(E) \otimes \mathfrak{N}(F)$ est l'ensemble des éléments de la forme $s^{-1} u$, où $u \in \mathfrak{U} \otimes \mathfrak{B}$ et où $s$ parcourt l'ensemble $S$ des éléments de la forme $x \otimes y$, où $x, y$ sont $\neq 0$ dans $\mathfrak{u}$ et $\mathfrak{B}$ respectivement. Il en résulte que tout idéal $\mathfrak{r}$ de $\mathfrak{H}(E) \otimes \mathfrak{R}(F)$ est engendré par son intersection avec $\mathfrak{H} \otimes \mathfrak{B}$; car, si $s^{-1} u \in \mathfrak{r}$, on a $u \in \mathfrak{r} \cap(\mathfrak{u} \otimes \mathfrak{B})$, et $s^{-1} \in \mathfrak{R}(E)$ $\otimes \mathfrak{A}(F)$. Les idéaux $\mathfrak{p}$ et $q \cap(\Re(E) \otimes \mathfrak{R}(F)$ ), ayant même intersection avec $\mathfrak{H} \otimes \mathfrak{B}$, sont identiques, ce qui démontre notre assertion. L'anneau $\mathfrak{o}$ ne dépend donc pas des choix de $U$ et de $V$. Soit $T_{1}$ une composante irréductible de $T$; on peut trouver des sous-variétés ouvertes affines $U$ de $E$ et $V$ de $F$ telles que $U \times V$ rencontre $T_{1}$. L'ensemble non vide $(U \times V) \cap T_{1}$ est relativement ouvert dans $T_{1}$, donc dense dans $T_{1} ; T_{1}$ est donc contenu dans l'adhérence de l'ensemble fermé irréductible $(U \times V) \cap T$; puisque $T_{1}$ est une composante irréductible de $T$, il est identique à l'adhérence de $(U \times V) \cap T$, et les fonctions de $\mathfrak{R}(E \times F)$ qui ont des traces sur $T_{1}$ sont les mêmes que celles qui ont des traces sur $(U \times V) \cap T$ : ce sont celles de l'anneau $\mathrm{D}$, qui ne dépend pas de $T_{1}$. Or, $T_{1}$ est fermé, et une partie irréductible fermée de $E \times F$ est déterminée de manière unique par la connaissance de l'anneau des fonctions qui ont des traces sur cette partie. On en conclut que $T$ n'a qu'une seule composante irréductible, donc que c'est une correspondance irréductible fermée. De plus, le corps $\Re(T)$ des fonctions sur $T$ est isomorphe à l'anneau $\mathfrak{o} / \mathfrak{q}$, et il est clair que l'homomorphisme 1 définit un isomorphisme de ce corps sur $\mathfrak{L} ; \mathfrak{R}(T)$ est donc isomorphe à \&. Il nous reste à montrer que $T$ n'est pas dégénérée; nous allons en fait établir un résultat beaucoup plus fort, à savoir le suivant: les projections sur 
$E$ et sur $F$ d'une partie ouverte non vide $T_{0}$ de $T$ contiennent des parties overtes non vides (donc denses) de $E$ et de $F$. Utilisant les mêmes notations que plus haut, nous poserons de plus $\mathfrak{u}^{\prime}=\lambda(\mathfrak{H}), \mathfrak{B}^{\prime}=\lambda^{\prime}(\mathfrak{W}), \mathfrak{W}^{\prime}=\Lambda(\mathfrak{H} \otimes \mathfrak{B}) ;$ ce sont des sous-anneaux de $\mathcal{L}$ dont chacun est engendré sur $\Omega$ par un nombre fini d'éléments; de plus, $\mathfrak{W}^{\prime}$ est engendré par $\mathfrak{u}^{\prime}$ et $\mathfrak{B}^{\prime}$. L'ensemble $T_{0}$ est l'intersection de $T$ avec une,partie ouverte $W$ de $E \times F$. Il y a un idéal $\mathfrak{w} \neq\{0\}$ de $\mathfrak{U} \otimes \mathfrak{B}$ tel que $W \cap(U \times V)$ soit l'ensemble des points de $U \times V$ dont les anneaux locaux sont les anneaux locaux d'idéaux maximaux de $\mathfrak{u} \otimes \mathfrak{B}$ ne contenant pas $\mathfrak{w}$. L'ensemble $T \cap(U \times V)$ est l'ensemble des points dont les anneaux locaux sont les anneaux locaux d'idéaux maximaux de $\mathfrak{u} \otimes \mathfrak{B}$ contenant $\mathfrak{p} \cap(\mathfrak{H} \otimes \mathfrak{B})$. Or, $T$ étant irréductible, $T_{0}$ rencontre $T \cap(U \times V)$; il y a donc un idéal maximal de $\mathfrak{U} \otimes \mathfrak{B}$ qui ne contient pas $\mathfrak{m}$ mais qui contient $\mathfrak{p} \cap(\mathfrak{U} \otimes \mathfrak{B})$; $\mathfrak{w}$ contient donc un élément $w$ n'appartenant pas à $\mathfrak{p}$. Posons $w^{\prime}=A(w)$; c'est un élément $\neq 0$ de $\mathfrak{W}^{\prime}$. Or, $\mathfrak{W}^{\prime}$ se déduit de $\mathfrak{u}^{\prime}$ par adjonction d'un nombre fini $\mathrm{d}^{\prime}$ éléments (par exemple, d'un ensemble de générateurs de $\mathfrak{V}^{\prime}$ sur $\Omega$ ). On sait que, dans ces conditions, il existe un élément $f^{\prime} \neq 0$ de $\mathfrak{u}^{\prime}$ qui possède la propriété suivante: tout homomorphisme de $\mathfrak{u}^{\prime}$ sur $\Omega$ qui coïncide avec l'identité sur $\Omega$ et qui n'applique pas $f^{\prime}$ sur 0 se prolonge d'au moins une manière en un homomorphisme de $\mathfrak{W}^{\prime}$ sur $\Omega$ qui n'applique pas $w^{\prime}$ sur 0 . Soit $f$ l'élément de $\mathfrak{u}$ tel que $\lambda(f)=f^{\prime}$, et soit $P$ un point de $U$ tel que $f(P) \neq 0$. Soit $\varphi$ l'homomorphisme $x \rightarrow x(P)$ de $\mathfrak{u}$ sur $\Omega ; \varphi \circ \lambda^{-1}$ est alors un homomorphisme de $\mathfrak{u}^{\prime}$ sur $\Omega$ qui se prolonge en un homomorphisme $\psi$ de $\mathfrak{B}^{\prime}$ sur $\Omega$ qui n'applique pas $w^{\prime}$ sur 0 . L'application $\psi \circ d$ est homomorphisme de $\mathfrak{U} \otimes \mathfrak{B}$ sur $\Omega$; son noyau $\mathfrak{R}$ est un idéal maximal de $\mathfrak{U} \otimes \mathfrak{B}$ qui contient le noyau $\mathfrak{p} \cap(\mathfrak{U} \otimes \mathfrak{B})$ de la restriction de $A$ à $\mathfrak{U} \otimes \mathfrak{B}$, mais qui ne contient pas $w$. Il en résulte que $\mathfrak{Y}$ ne contient pas $\mathfrak{w}$; l'anneau local de $\mathfrak{N}$ est donc l'anneau local d'un point de $T_{0}$. La restriction de $\psi \circ A$ à $\mathfrak{u}$ étant $\varphi$, il est clair que la projection de ce point est $P$. On voit donc que tout point $P$ de $U$ tel que $f(P) \neq 0$ appartient à la projection de $T_{0}$ sur $E$, ce qui montre que cette dernière contient une partie ouverte non vide de $E$. On voit de même que sa projection sur $F$ contient une partie ouverte non vide de $F$.

Supposons qu'une autre extension composée $\left(\mathfrak{R}_{1}, \lambda_{1}, \lambda_{1}^{\prime}\right)$ définisse la même correspondance $T$. Les homomorphismes $\lambda_{1}$, $\lambda_{1}^{\prime}$ définissent un homomorphisme $A_{1}$ de $\Re(E) \otimes \Re(F)$ dans $\Omega_{1}$. Les noyaux de $A$ et $A_{1}$ sont tous deux identiques 
à l'ensemble des fonctions de $\Re(E) \otimes \Re(F)$ de trace nulle sur $T$. On en déduit qu'il y a un isomorphisme $\theta$ de $A(\Re(E) \otimes \Re(F))$ sur $\Lambda_{1}(\Re(E) \otimes \Re(F))$ tel que $\theta \circ A=A_{1}$. Cet isomorphisme se prolonge en un isomorphisme, que nous désignons encore par $\theta$, de $\&$ sur $\Omega_{1}$, et il est clair que $\lambda_{1}=\theta \circ \lambda, \lambda_{1}^{\prime}=\theta \circ \lambda^{\prime}$, ce qui montre que les extensions composées $\left(\mathscr{L}, \lambda, \lambda^{\prime}\right)$ et $\left(\Omega_{1}, \lambda_{1}, \lambda_{1}^{\prime}\right)$ sont isomorphes. Réciproquemenf, il est évident que des extensions composées isomorphes définissent la même correspondance.

L'ensemble $T$ a été défini comme l'ensemble des couples $(P, Q)$ tels que $\mathfrak{p} \cap(\mathfrak{D}(P) \otimes \mathfrak{p}(Q)) \subset \mathrm{m}_{P, Q} . \quad$ Si cette condition est satisfaite, l'anneau local $\mathfrak{o}(P, Q)$ de $\mathfrak{m}_{P, Q}$ est contenu dans le domaine de définition $\mathfrak{o}$ de l'homomorphisme .1(rappelons que $\mathfrak{D}$ est l'anneau local de l'ideal $\mathfrak{p}$ de $\Re(E) \otimes \mathfrak{R}(F)) ; \lambda(\mathfrak{D}(P))$ et $\lambda^{\prime}(\mathfrak{D}(Q)$ ) sont contenus dans $A(\mathfrak{D}(P, Q))$, et les idéaux maximaux $\lambda\left(\mathfrak{m}_{P}\right), \lambda^{\prime}\left(\mathrm{m}_{Q}\right)$ de ces anneaux sont contenus dans l'idéal maximal $A\left(\mathfrak{m}_{P, Q} \mathfrak{D}(P, Q)\right)$ de $A(\mathfrak{o}(P, Q))$; il en résulte que $\lambda(\mathfrak{D}(P))$ et $\lambda^{\prime}(\mathfrak{D}(Q))$ se correspondent. Supposons maintenant que la condition ne soit pas satisfaite. Comme $m_{P, Q}$ est un idéal maximal de $\mathfrak{o}(P)$ $\otimes \mathfrak{o}(Q)$, l'idéal $\mathrm{m}_{P, Q}+\mathfrak{p} \cap(\mathfrak{o}(P) \otimes \mathfrak{o}(Q))$ contient alors 1 . Puisque $A$ applique les éléments de $p$ sur $0, A\left(\mathfrak{m}_{P, Q}\right)$ contient 1 . Or on a $\mathfrak{m}_{P, Q}=\mathfrak{m}_{P} \otimes \mathfrak{o}(Q)+\mathfrak{p}(P)$ $\otimes \mathfrak{m}_{Q}$; l'idéal engendré par $\lambda\left(\mathfrak{m}_{P}\right)$ et $\lambda^{\prime}\left(\mathfrak{m}_{Q}\right)$ dans l'anneau engendré par $\lambda(\mathfrak{D}(P))$ et $\lambda^{\prime}(\mathfrak{D}(Q))$ contient donc 1 , et $\lambda(\mathfrak{D}(P)), \lambda^{\prime}(\mathfrak{D}(Q))$ ne se correspondent pas.

Reste à montrer que toute correspondance irréductible fermée non dégénérée $T$ entre $E$ et $F$ peut se définir au moyen d'une extension composée convenable. Soient 0 l'anneau des fonctions de $\Re(E \times F)$ qui ont des traces sur $T$ et $\mathfrak{q}$ l'idéal de $\mathfrak{D}$ composé des fonctions de trace $0 ; \mathfrak{L}=\mathfrak{b} / \mathfrak{q}$ est donc un corps isomorphe à $\Re(T)$. L'idéal q n'a que 0 en commun avec $\Re(E) \otimes 1$. Soit en effet $f$ une fonction $\neq 0$ de $\Re(E)$. La projection de $T$ sur $E$, qui est dense, recontre l'ensemble ouvert non vide des points de $E$ où $f$ et $f^{-1}$ sont toutes deux définies: il y a donc un point $(P, Q) \in T$ tel que $f$ soit défini en $P$ et y soit $\neq 0 ; f \otimes 1$

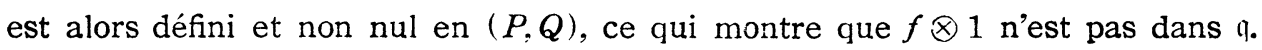
On en déduit que l'homomorphisme canonique de $\mathfrak{b}$ sur $\mathfrak{v} / \mathfrak{q}=\mathfrak{Q}$ définit un isomorphisme $\lambda$ de $\mathfrak{R}(E)$ sur un sous-corps de $\&$ qui applique tout $f \in \mathfrak{M}(E)$ sur la classe de $f \otimes 1$ modulo q. On définit de manière analogue un isomorphisme $\lambda^{\prime}$ de $\mathfrak{R}(F)$ sur un sous-corps de $\mathfrak{2}$. Toute fonction $u$ sur $E \times F$ qui a une trace sur $T$ peut d'ailleurs se mettre. sous la forme du quotient de deux fonctions appartenant à $\mathfrak{R}(E) \otimes \mathfrak{R}(F)$, le dénominateur n'étant pas dans q. Soit en effet 
$(P, Q)$ un point de $T$ en lequle $u$ est définie; $u$ appartient alors à $\mathfrak{D}(P, Q)$ qui est l'anneau local de l'idéal $\mathfrak{m}_{P, Q}=\mathfrak{m}_{P} \otimes \mathfrak{D}(Q)+\mathfrak{D}(P) \otimes \mathfrak{M}_{Q}$, de $\mathfrak{D}(P) \otimes \mathfrak{D}(Q)\left(\mathfrak{m}_{P}\right.$ et $\mathrm{m}_{Q}$ étant les idéaux maximaux de $\mathfrak{o}(P)$ et $\left.\mathfrak{o}(Q)\right)$. Puisque $(P, Q) \in T$, on a $q \cap(\mathfrak{o}(P) \otimes \mathfrak{o}(Q)) \subset \mathrm{m}_{P, Q} ;$ la fonction $u$ se représente comme quotient de deux éléments de $\mathfrak{o}(P) \otimes \mathfrak{o}(Q)$, avec un dénominateur n'apprtenant pas à $\mathfrak{m}_{P, Q}$, donc n'appartenant pas à q. Il résulte tout de suite de là que le corps $\mathbb{L}$ est engendré par $\lambda(\Re(E))$ et $\lambda^{\prime}(\Re(F))$, donc que $\left(\mathcal{R}, \lambda, \lambda^{\prime}\right)$ est une extension composée de $\Re(E) / \Omega$ et $\Re(F) / \Omega$. Il est clair que cette extension composée définit la correspondance $T$. Le théorème 2 est donc démontré.

On notera que la démonstration a établi que les projections de toute partie ouverte non vide de $T$ sur $E$ et sur $F$ contiennent des parties ouvertes non vides de ces variétés.

III.

Soit $E$ une variété. On appelle constructible toute partie de $E$ qui peut se représenter comme réunion d'un nombre fini de sous-variétés de $E$.

Proposition 5. Pour qu'une partie de E soit constructible, il faut et suffit qu'elle puisse se représenter comme réunion d'un nombre fini d'ensembles dont chacun est l'intersection d'une partie fermée et d'une partie overte de $E$.

Une sous-variété de $E$ est relativement ouverte dans son adhérence, donc est l'intersection d'un ensemble fermé et d'un ensemble ouvert; la condition est donc nécessaire. Pour montrer qu'elle est suffisante. il suffit de montrer que l'intersection d'une partie fermée $A$ et d'une partie ouverte $U$ est constructible. Les composantes irréductibles $A_{1}, \ldots, A_{h}$ de $A$ sont des sous-variétés fermées de $E$, et $A \cap U$ est la réunion des $A_{i} \cap U$; chaque $A_{i} \cap U$; est ou bien vide ou bien une sous-variété.

Proposition 6. La réunion et l'intersection de deux parties constructibles de $E$ sont constructibles.

Cela résulte immédiatement de la proposition 5 .

PROPOSITION 7. Les composantes irréductibles d'une partie constructible de E sont constructibles.

En effet, si $A$ est constructible, les composantes irréductibles de $A$ sont 
relativement fermées dans $A$, et sont par suite les intersections de $A$ avec des ensembles fermés, donc constructibles.

Proposition 8. Soit A une partie de E. Supposons que, pour chaque point $P_{0}$ de $A$ il $y$ ait une partie ouverte $U$ de $E$ et des fonctions $f_{l}, \ldots, g_{m}, g_{1}, \ldots, g_{n}$ sur $E$, definies en tout point de $U$, telles que $A \cap U$ soit l'ensemble des points $P$ de Utels que l'on ait $f_{1}(P)=0, \ldots, f_{m}(P)=0, g_{1}(P) \neq 0, \ldots, g_{n}(P) \neq 0 ; A \in s t$ alors constructible.

Désignons par $U\left(P_{0}\right)$ un ensemble ouvert ayant la propriété requise. L'ensemble $A \cap U\left(P_{0}\right)$ est l'intersection d'une partie ouverte de $U\left(P_{0}\right)$ avec une partie relativement fermée de cet ensemble; il est donc constructible. Par ailleurs, la famille des réunions finies d'ensembles de la forme $U\left(P_{0}\right)$ admet un élément maximal $U$; il est clair que $A \subset U$, donc que $A$ est la réunion d'un nombre fini des ensembles $A \cap U\left(P_{0}\right)$.

THÉoRÈme 3. Soient $T$ une correspondance irréductible entre une variété $E$ et une variété $F$ et $B$ une partie constructible de $F$. L'ensemble des points de $E$ qui correspondent par $T$ à des points de. $B$ est alors constructible.

Nous procéderons par récurrence sur la somme $n$ des dimensions de $E$ et de $F$. Le théorème est évident si $n=0$. Supposons que $n>0$ et que le théorème soit vrai pour les couples de variétés dont la somme des dimensions est $<n$. Supponsons d'abord la correspondance $T$ dégénérée. Ses projections sur $E$ et sur $F$ sont irréductibles; leurs adhérences $E^{\prime}, F^{\prime}$ sont donc des sous-variétés fermées de $E, F$, et on a ou bien $E^{\prime} \neq E$ ou bien $F^{\prime} \neq F$; dans les deux cas, la somme des dimensions de $E^{\prime}$ et de $F^{\prime}$ est $<n$. L'ensemble $B \cap F^{\prime}$ est une partie constructible de $F^{\prime}$; l'ensemble $A$ des points de $E$ qui correspondent à des points de $B$ est aussi l'ensemble des points de $E^{\prime}$ qui correspondent à des points de $B \cap F^{\prime}$; il est par suite constructible dans $E^{\prime}$, donc aussi dans $E$. Supposons maintenant $T$ non dégénérée. Il est clair qu'il suffit de démontrer le théorème dans le cas où $B$ est une sous-variété. Supposons d'abord que l'adhérence $\bar{B}$ de $B$ soit $\neq F$. Nous désignerons par $\pi_{F}$ et $\pi_{F}$ les projections de $E \times F$ sur $E$ et sur $F$. L'ensemble $\pi_{F}^{-1}(\bar{B}) \cap T$ es t relativement fermé dans $T$; ses composantes irréductibles sont des correspondances irréductibles $T_{1}, \ldots$ ., $T_{h}$ toutes dégénérées. L'ensemble des points de $E$ qui correspondent à des points de $B$ par $T$ est la réunion des $A_{i}(i=1, \ldots, h)$, où $A_{\imath}$ est l'ensemble des 
points de $E$ qui correspondent à des points de $B$ par $T_{i}$ : cet ensemble est donc constructible. Supposons maintenant que $B$ soit dense dans $F$. Cet ensemble est alors ouvert, et $T_{0}=T \cap \pi_{F}^{-1}(B)$ est une partie ouverte, d'ailleurs non vide (puisque la projection de $T$ sur $F$ est dense dans $F$ ) de $T$ : c'est donc une correspondance irréductible entre $E$ et $B$, et on est ramené au cas où $B=F$. S’il en est ainsi, l'ensemble considéré est la projection de $T$ sur $E$. L'adhérence $\bar{T}$ de $T$ est une correspondance irréductible fermée, que l'on peut supposer non dégénérée, et $T$ est une partie relativement ouverte de $\bar{T}$. Nous avons vu qu'il en résulte que la projection de $T$ sur $E$ contient une partie ouverte $E_{0}$ non vide de $E$. Le complémentaire de $E_{0}$ par rapport à $E$ se décompose en un nombre fini de sous-variétés fermées $C_{i}(1 \leqq i \leqq m)$; lensemble $T \subset \pi_{E}^{-1}\left(C_{i}\right)$ est relativement fermé dans $T$; s'il n'est pas vide, il se représente comme réunion d'un nombre fini de sous-variétés $T_{i j}(j=1, \ldots, r(i))$. La projection de $T$ sur $E$ est la réunion de $E_{0}$ et des projections des $T_{i j}$. Or les $T_{i j}$ sont des correspondances irréductibles dégénérées; leurs projections sont donc constructibles, et il en est de même de la projection de $T$.

Corollaire. Soient $E$ et $F$ des variétés, et $C$ une partie constructible de $E \times F$. Les projections de $C$ sur $E$ et sur $F$ sont alors constructibles.

Il suffit de considérer le cas où $C$ est une sous-variété de $E \times F$, donc une correspondance irréductible entre $E$ et $F$; dans ce cas, le corollaire résulte immédiatement du théorème 3 .

\section{IV.}

Nous appellerons admissibles les sous-corps $K$ du domaine fondamental $\Omega$ tels que $\Omega$ soit degré de transcendance infini sur $K$. On appelle structure de $K$-variété sur un ensemble $E$ une structure constituée par les données d'une structure de variété sur $E$ et d'un sous-corps $\Re_{K}(E)$ de $\Re(E)$ qui possède les propriétés suivantes: $\Re_{K}(E)$ contient $K$; les extensions $\Omega / K$ et $\mathfrak{R}_{K}(E) / K$ sont linéairement disjointes sur $K$, et on a $\Re(E)=\Omega\left(\Re_{K}(E)\right)$. La structure de variété qui intervient dans la définition d'une structure de $K$-variété s'appelle la structure de variété sous-jacente à la structure considérée de $K$-variété. Définir sur une variété $E$ une structure de $K$-variété, ce sera, par définition, définir une $K$-variété dont la variété donnée soit la variété sous-jacente. Si $E$ est une $K$ variété, les fonctions de $\mathfrak{R}_{K}(E)$ sont dites "définies sur $K$ ”. 
Si $E$ est une $K$-variété et $K^{\prime}$ un sous-corps admissible de $\Omega$ contenant $K$, soit $\Re_{K^{\prime}}(E)$ le corps $K^{\prime}\left(\Re_{K}(E)\right)$; les fonctions de ce corps sont dites être "définies sur $K^{\prime \prime}$. Il est clair que les extensions $\Omega / K^{\prime}, \Re_{K^{\prime}}(E) / K^{\prime}$ sont linéairement disjointes et que $\Re(E)=\Omega\left(\Re_{K^{\prime}}(E)\right)$; le corps $\Re_{K^{\prime}}(E)$ définit donc sur $E$ une structure de $K^{\prime}$-variété, dont on déduit qu'elle se déduit de la structure donnée de $K$-variété par extension à $K^{\prime}$ du corps de base.

Nous dirons qu'une structure de $K$-variété définie par un corps $\Re_{K}(E)$ est "forte" si l'on peut représenter $E$ comme réunion d'un nombre fini de variétés affines $V_{1}, \ldots, V_{h}$ telles que, pour chaque $i$, l'anneau affine de $V_{i}$ soit engendré par adjonction (d'anneau) à $\Omega$ d'un certain nombre (fini) de fonctions défines sur $K$.

Montrons que, pour toute variété $E$, il existe des sous-corps admissibles $K$ de $\Omega$ tels que $E$ puisse être munie d'une structure de $K$-variété forte. Représentons d'une manière quelconque $E$ comme réunion d'un nombre fini de variétés affines $V_{i}(i \leqq 1, \ldots, h)$, et soit $\mathfrak{B}_{\imath}$ l'anneau affine de $V_{i}$. Mettons $\mathfrak{B}_{i}$ sous la forme $\Omega\left[f_{i 1}, \ldots, f_{i, n(i)}\right]$, où les $f_{i, j}$ sont des fonctions sur $E$. Il existe alors un homomorphisme $\theta_{i}$ de l'anneau $\Omega\left[X_{1}, \ldots, X_{n(i)}\right]$ des polynômes en $n$ lettres à coefficients dans $\Omega$ sur $\mathfrak{B}_{i}$ qui coïncide avec l'identité sur $\Omega$ et qui applique $X_{j}$ sur $f_{i j}(1 \leqq j \leqq n(i))$. Le noyau $\Re_{i}$ de cet homomorphisme est un idéal premier; on sait qu'il existe un corps $K_{i}$ engendré (au sens absolu) par un nombre fini d'éléments de $\Omega$ tel que $\mathfrak{P}_{i}$ ait un système de générateurs composé de polynômes à coefficients dans $K_{i}$; de plus, si $K_{i}$ est un corps quelconque satisfaisant à cette condition, les extensions $2 / K_{i}, K_{i}\left(f_{i, 1}, \ldots, f_{i, n(i)}\right)$ sont linéairement disjointes. En effet, ces extensions sont manifestement algébriquement disjointes l'une de l'autre, et on sait que la propriété énoncée du corps $K_{i}$ entraîne que $K_{i}\left(f_{11}, \ldots, f_{i, n(i)}\right) / K_{i}$ est une extensiun régulière. Il existe un corps $K$ engendré par un nombre fini d'élements qui contient tous les corps $K_{i}$ et qui est tel que les $h$ corps $K\left(f_{i 1}, \ldots, f_{i, n(i)}\right)(i=1, \ldots, h)$ soient identiques les uns aux autres. Exprimons en effet les $f_{i j}(1 \leqq j \leqq n(i))$ comme fractions rationnelles à coefficients dans $\Omega$ en les $f_{i^{\prime} j^{\prime}}\left(j^{\prime}=1, \ldots, n\left(i^{\prime}\right)\right)$, et ceci pour tous les couples $\left(i, i^{\prime}\right)$ d'indices distincts entre 1 et $h$; il suffit alors de prendre pour $K$ le corps engendré par tous les $K_{i}(1 \leqq i \leqq h)$ et par les coefficients des fractions rationnelles que l'on obtient. Il est alors clair que le corps $\Re_{K}(E)$ $=K\left(f_{i 1}, \ldots, f_{i, n(i)}\right)$ définit sur $E$ une structure de $K$-variété forte. 
Soit $E$ une $K$-variété. Nous appellerons $K$-topologie sur $E$ la topologie engedrée par les ensembles $D(f)$, où $f$ parcourt l'ensemble des fonctions définies sur $K$. Il est clair que cette topologie est moins fine que la topologie de Zariski sur $E$ (et, en général, strictement moins fine), c'est-à-dire que les ensembles ouverts (ou fermés) de la $K$-topologie sont aussi ouverts (ou fermés) dans la topologie de Zariski. Il résulte de là que l'espace $E$, muni de sa $K$-topologie, est un espace de Zariski. L'application identique de $E$, muni de sa $K$-topologie, dans $E$, muni de sa topologie de Zariski, étant continue, tout ensemble irréductible pour la $K$-topologie est aussi irréductible dans la topologie de Zariski ; la réciproque n'est en général pas vraie.

Si $K^{\prime}$ est un sur-corps admissible de $K$, on appelle $K^{\prime}$-topologie celle déduite de la structure de $K^{\prime}$-variété sur $E$ déduite de sa structure de $K$-variété par extension du corps de base. Cette topologie est plus fine que la $K$-topologie.

Nous dirons qu'un point $P^{\prime}$ de $E$ est une spécialisation d'un point $P$ par rapport à $K$ si $P^{\prime}$ appartient à l'adhérence de l'ensemble $\{P\}$ (réduit au point $P$ ) dans la $K$-topologie. Il est clair que $P^{\prime}$ est alors aussi spécialisation de $P$ par rapport à tout sur-corps (admissible) de $K$.

Proposition 9. Pour quiun point $P^{\prime}$ soit spécialisation dun point $P$ par rapport à $K$, il faut et suffit que toute fonction sur $E$ définie sur $K$ et definie en $P^{\prime}$ soit définie en $P$; cela entraîne que que toute fonction définie sur $K$ et définie en $P^{\prime}$ qui prend la valeur 0 en $P$ prend la valeur 0 en $P^{\prime}$.

Supposons que $P^{\prime}$ soit spécialisation de $P$. Soit $f$ une fonction définie sur $K$; si $f$ est définie en $P^{\prime}$, on a $P^{\prime} \in D(f)$. Or, $D(f)$ est ouvert dans la $K$ topologie; puisque $P^{\prime}$ est adhérent à $\{P\}, D(f)$ rencontre $\{P\}$ et $P \in D(f)$. Supposons réciproquement la condition satisfaite. Tous les ensembles de la forme $D(f)$, pour $f \in \Re_{K}(E)$, qui contiennent $P^{\prime}$ contiennent aussi $P$; il en résulte immédiatement que tout ensemble ouvert de la $K$-topologie qui contient $P^{\prime}$ contient aussi $P$, donc que $P^{\prime}$ est adhérent à $\{P\}$. Si une fonction $f \in \Re_{K}(E)$ est définie en $P^{\prime}$ et si $f(P)=0$, la fonction $f^{-1}$ (si $f \neq 0$ ) n'est pas définie en $P$, donc ne l'est pas non plus en $P^{\prime}$ (si $P^{\prime}$ est une spécialisation de $P$ ), ce qui entraîne que $f\left(P^{\prime}\right)=0$.

Soit $P$ un point quelconque de $E$. Considérons l'anneau $\mathfrak{D}(P) \cap \mathfrak{R}_{K}(E)$ des fonctions définies sur $K$ qui sont définies en $P$. L'application $f \rightarrow f(P)$ défini t 
un homomorphisme de cet anneau dans $\Omega$, et l'image de $\mathcal{D}(P) \cap \Re_{\kappa}(E)$ par cet homomorphisme est manifestement un sous-corps de $\Omega$ contenant $K$; on désigne ce corps par $K(P)$. Supposons que $P^{\prime}$ soit une spécialisation de $P$ par rapport à $K$. L'anneau $\mathfrak{o}\left(P^{\prime}\right) \cap \mathfrak{A}_{K}(E)$ est alors contenu dans $\mathfrak{v}(P) \cap \mathfrak{X}_{K}(E)$; son image dans $K(P)$ est un sous-anneau de $K(P)$, appelé anneau de la spécialisation $P \rightarrow P^{\prime}$; il y a un homomorphisme de cet anneau sur $K\left(P^{\prime}\right)$ qui applique $f(P)$ sur $f\left(P^{\prime}\right)$ pour toute fonction $f \in \Re_{K}(E)$ qui est définie en $P^{\prime}$.

Lorsque la structure de K-variété de E est une structure forte, là sconnaissance de l'anneau $\mathrm{o}(P) \cap \mathfrak{H}_{\mathrm{K}}(E)$ et de l'homomorphisme $f \rightarrow f(P)$ de cet anneau sur $K(P)$ determine entièrement le point $P$. Le point $P$ est alors en effet contenu dans une sous-variété affine ouverte $V$ de $E$ dont lanneau affine $\mathfrak{B}$ est engendré sur $\Omega$ par des éléments de $\mathfrak{R}_{K}(E)$. Si $P^{\prime}$ est un point de $E$ tel que $\mathfrak{n}\left(P^{\prime}\right) \cap \mathfrak{\Re}_{K}(E)=\mathfrak{D}(P) \cap \mathfrak{H}_{K}(E), \mathfrak{p}\left(P^{\prime}\right)$ contient l'anneau $\mathfrak{B} \cap \mathfrak{H}_{K}(E)$, donc aussi $\mathfrak{B}$; il en résulte, comme on le sait, que $P^{\prime}$ appartient à $V$. Si les homomorphismes $f \rightarrow f(P)$ et $f \rightarrow f\left(P^{\prime}\right)$ de $\mathfrak{o}(P) \cap \mathfrak{T}_{K}(E)$ sont identiques, toutes les fonctions de $\mathfrak{B}_{K}$ prennent la même valeur en $P$ et en $P^{\prime}$; il en est donc de même de toute fonction de $\mathfrak{B}$, d'où $P=P^{\prime}$. Par contre, notre conclusion tomberait en défaut si nous ne faisions pas l'hypothèse que la structure de $K$-variété de $E$ est forte. Indiquons comment on peut en construire un exemple, sans entrer dans les détails. Partons du plan affine $E_{0}$; soit $K$ un sous-corps admissible quelconque de $\Omega$, et soit $\Re_{K}\left(E_{0}\right)$ le corps obtenu par adjonction à $K$ des fonctions coordonnées sur $E_{0}$; la donnée de ce corps définit sur $E_{0}$ une structure de $K$-variété. Soit maintenant $E$ une variété qui se déduit de $E_{0}$ en transformant en une courbe un point $P_{0}$ de $E_{0}$ dont les coordonnées $a$ et $b$ sont algébriquement indépendantes par rapport à $K$. On a donc $\Re(E)=\Re\left(E_{0}\right)$ (à un isomorphisme près), et la structure de $K$-variété de $E_{0}$ définit une structure de $K$-variété de $E$. Soit $P$ un point quelconque de la courbe $C$ qui correspond à $P_{0}$; les fonctions coordonnées $x$ et $y$ sur $E_{0}$ sont alors définies en $P$ et $y$ prennent les valeurs $a$ et $b$. Il est clair que toute fonction $f$ de $\Re_{K}(E)$ est définie en $P_{0}$ (en tant que fonction sur $E_{0}$ ) et en $P$ (en tant que fonction sur $E$ ) et que $f(P)=f\left(P_{0}\right)$. On a donc $\mathfrak{o}(P) \cap \Re_{K}(E)=\Re_{K}(E)$, et $f(P)=f\left(P_{0}\right)$ pour tout $f \in \Re_{K}(E)$; les fonctions de $\Re_{K}(E)$ prennent par suite toutes la même valeur en deux points quelconques de $C$.

Il serait donc peut-être préférable de n'admettre que les structures de $K$ - 
variétés qui sont fortes au sens précédemment défini. Cependant, nous allons voir que certains résultats sont valables pour les structures de $K$-variétés quelconques.

Proposition 10. Pour qu'une partie de E fermée dans la K-topologie soit irréductible, il faut et suffit qu'elle se compose de toutes les spécialisations (par rapport à $K$ ) d'un même point $P$.

L'ensemble $S(P)$ des spécialisations d'un point $P$ est l'adhérence de $\{P\}$ dans la $K$-topologie, donc est fermé. L'une au moins des composantes irréductibles (dans la $K$-topologie) de $S(P)$, soit $S_{1}(P)$, contient $P$; l'ensemble $S_{1}(P)$, étant fermé, contient alors l'adhérence $S(P)$ de $\{P\}$, ce qui montre que $S(P)$ est irréductible. Soit réciproquement $A$ une partie irréductible de $E$ (dans la $K$-topologie). L'ensemble $A$ se représente alors comme réunion d'ensembles $A_{i}(1 \leqq i \leqq h)$ irréductibles dans la topologie de Zariski; $A$ étant fermé dans la topologie de Zariski, il en est de même des $A_{i}$, qui sont des sous-variétés de $E$. Soit $V_{i}$ une sous-variété affine ouverte de $E$ qui rencontre $A_{i}$, et soit $\mathfrak{B}_{i}$ son anneau affine. Les fonctions de $\mathfrak{B}_{i}$ de trace nulle sur $A_{i}$ forment un idéal premier $\mathfrak{p}_{i}$ de $V_{i}$. On peut trouver un corps $K^{\prime}$ qui se déduit de $K$ par adjonction d'un nombre fini d'éléments et qui possède les propriétés suivantes: il existe des fonctions $f_{1}, \ldots$ , $f_{n}$ de $\mathfrak{R}_{K^{\prime}}(E)$ telles que $\mathfrak{B}_{i}=\Omega\left[f_{1}, \ldots, f_{n}\right]$ et des fonctions $u_{1}, \ldots, u_{m}$ de $\mathfrak{R}_{K},(E)$ qui forment un ensemble de générateurs de l'idéal $\mathfrak{p}_{i}$. Posons $\mathfrak{B}_{i}^{\prime}=\mathfrak{B}_{i}$ $\cap \mathfrak{R}_{K^{\prime}}(E), \mathfrak{p}_{i}^{\prime}=\mathfrak{p}_{i} \cap \mathfrak{V}_{i}^{\prime} ; \mathfrak{p}_{i}^{\prime}$ est alors un idéal premier de $\mathfrak{B}_{i}^{\prime}$ et $\mathfrak{B}_{i}^{\prime}=K^{\prime}\left[f_{1}, \ldots\right.$, $f_{n}$ ]. Le corps des quotients $L$ de l'anneau $\mathfrak{B}_{i}^{\prime} / \mathfrak{p}_{i}^{\prime}$ contient $K^{\prime}$ comme sous-corps et $L / K^{\prime}$ est une extension de type fini. Il y a donc un isomorphisme $\varphi$ de $L$ sur un sous-corps de $\Omega$ qui coïncide avec l'identité sur $K^{\prime}$ (car $\Omega$ est algébriquement clos et de degré de transcendance infini sur $K^{\prime}$ ). Cet isomorphisme, composé avec l'application canonique de $\mathfrak{B}_{i}^{\prime}$ sur $\mathfrak{B}_{i}^{\prime} / \mathfrak{p}_{i}^{\prime}$, donne un homomorphisme $\psi^{\prime}$ de $\mathfrak{2}_{i}^{\prime}$ dans $\Omega$. Puisque $K^{\prime}$ contient $K$, les structures d'algèbres sur $K^{\prime}$ de $\mathfrak{V}_{i}^{\prime}$ et de $\Omega$ sont linéairement disjointes, et $\psi^{\prime}$ se prolonge en un homomorphisme $\psi$ de $\mathfrak{B}_{i}$ dans $\Omega$. Le noyau de $\psi$ est un idéal maximal $\mathfrak{M}_{i}$ de $V_{i}$ dont l'anneau local est celui d'un point $P_{i}$ de $V_{i}$. Il est clair que $\psi\left(u_{i}\right)=0(1 \leqq i \leqq m)$, d'où $\mathfrak{p}_{i} \subset \mathfrak{M}$ et $P_{i} \in A_{i}$. Tout point $P_{i}^{\prime}$ de $V_{i} \cap A_{i}$ est une spécialisation de $P_{i}$ par rapport à $K^{\prime}$. Soit en effet $f$ une fonction de $\Re_{K^{\prime}}(E)$ définie en $P_{i}^{\prime}$; elle se met sous la forme $A\left(f_{1}, \ldots, f_{n}\right) / B\left(f_{1}, \ldots, f_{n}\right)$, où $A$ et $B$ sont des polynômes à 
coefficients dans $\Omega$ et où $B\left(f_{1}\left(P_{i}^{\prime}\right), \ldots, f_{n}\left(P_{i}^{\prime}\right)\right) \neq 0$. Soit $\left(\omega_{j}\right)_{j \in J}$ une base de $\Omega / K^{\prime}$; on peut écrire $A=\sum_{j \in J} \omega_{j} A_{j}, B=\sum_{j \in J} \omega_{j} B_{j}$, où les $A_{j}, B_{j}$ sont des polynômes à coefficients dans $K^{\prime}$. On a $\sum_{j \in J} \omega_{j}\left(A_{j}\left(f_{1}, \ldots, f_{n}\right)-f B_{j}\left(f_{1}, \ldots, f_{n}\right)\right)=0$; les $\omega_{j}$ étant linéairement indépendants sur $K^{\prime}\left(f_{1}, \ldots, f_{n}\right)$, on a $A_{j}\left(f_{1}, \ldots, f_{n}\right)$ $=f B_{j}\left(f_{1}, \ldots, f_{n}\right)$ pour tout $j$. Or, il existe au moins un $j$ tel que $B_{j}\left(f_{1}\left(P_{i}^{\prime}\right)\right.$, $\left.\ldots, f_{n}\left(P_{i}^{\prime}\right)\right) \neq 0$; on voit donc que l'on peut supposer sans restriction de généralité que $A$ et $B$ sont à coefficients dans $K^{\prime}$. Puisque $P_{i}^{\prime}$ appartient à $A_{i}$, toute fonction de $p_{i}^{\prime}$ prend la valeur 0 en $P_{i}^{\prime}$, d'où $B\left(f_{1}, \ldots, f_{n}\right) \notin p_{i}^{\prime}$. Il en résulte immédiatement que $B\left(f_{1}\left(P_{i}\right), \ldots, f_{n}\left(P_{i}\right)\right) \neq 0$, donc que $f$ est définie en $P_{i}: P_{i}^{\prime}$ est une spécialisation de $P_{i}$ par rapport à $K^{\prime}$, donc a fortiori par rapport à $K$. Soit $S\left(P_{i}\right)$ lensemble des spécialisations de $P_{i}$ par rapport à $K$; $S\left(P_{i}\right)$ est donc fermé dans la $K$-topologie, et par suite aussi dans la topologie de Zariski. L'ennsemble $V_{i} \cap A_{i}$ étant dense dans $A_{i}, S\left(P_{i}\right)$ contient $A_{i}$. L'ensemble $A$ est donc la réunion des $S\left(P_{i}\right)(i=1, \ldots, h)$; étant irréductible, il coïncide avec l'un des $S\left(P_{i}\right)$, ce qui démontre la proposition 10.

Il est d'ailleurs facile de montrer, par le raisonnement au moyen duquel nous avons établi l'existence de structures fortes, que, pour toute sous-variété $A$ de $E$, il existe des corps admissibles $K^{\prime}$ contenant $K$ tels que $A$ soit fermé dans la $K^{\prime}$-topologie: on appelle alors point générique de $A$ par rapport à $K^{\prime}$ tout point de $A$ tel que $A$ soit l'ensemble des spécialisations de ce point par rapport à $K^{\prime}$.

\section{BIBLIOGRAPHIE}

[W] A. Weil, Foundations of algebraic Geometry. Amer. Math. Soc. Colloquium public., 1946. 